\title{
Aliphatic Amines in Antarctic CR2, CM2, and CM1/2 Carbonaceous Chondrites
}

José C. Aponte, ${ }^{\text {a,b,* Hannah L. McLain, }},{ }^{\text {a,b }}$ Jason P. Dworkin, ${ }^{\text {a }}$ and Jamie E. Elsila ${ }^{a}$

a Solar System Exploration Division, Code 691, NASA Goddard Space Flight Center, Greenbelt, Maryland 20771, USA.

${ }^{\mathrm{b}}$ Department of Chemistry, Catholic University of America, Washington, DC 20064, USA.

* Corresponding author:

jose.c.aponte@nasa.gov; tel.: +1.301.614.6916; fax: +1.301.286.1683. 


\begin{abstract}
Meteoritic water-soluble organic compounds provide a unique record of the processes that occurred during the formation of the solar system and the chemistry preceding the origins of life on Earth. We have investigated the molecular distribution, compound-specific $\delta^{13} \mathrm{C}$ isotopic ratios and enantiomeric compositions of aliphatic monoamines present in the hot acid-water extracts of the carbonaceous chondrites LAP 02342 (CR2), GRA 95229 (CR2), LON 94101 (CM2), LEW 90500 (CM2), and ALH 83100 (CM1/2). Analyses of the concentration of monoamines in these meteorites revealed: a) the CR2 chondrites studied here contain higher concentrations of monoamines relative to the analyzed CM2 chondrites; b) the concentration of monoamines decreases with increasing carbon number; and c) isopropylamine is the most abundant monoamine in these CR2 chondrites, while methylamine is the most abundant amine species in these CM2 and CM1/2 chondrites. The $\delta^{13} \mathrm{C}$ values of monoamines in CR2 chondrite do not correlate with the number of carbon atoms; however, in $\mathrm{CM} 2$ and $\mathrm{CM} 1 / 2$ chondrites, the ${ }^{13} \mathrm{C}$ enrichment decreases with increasing monoamine carbon number. The $\delta^{13} \mathrm{C}$ values of methylamine in CR2 chondrites ranged from -1 to $+10 \%$, while in $\mathrm{CM} 2$ and $\mathrm{CM} 1 / 2$ chondrites the $\delta^{13} \mathrm{C}$ values of methylamine ranged from +41 to $+59 \%$. We also observed racemic compositions of sec-butylamine, 3-methyl2-butylamine, and sec-pentylamine in the studied carbonaceous chondrites. Additionally, we compared the abundance and $\delta^{13} \mathrm{C}$ isotopic composition of monoamines to those of their structurally related amino acids. We found that monoamines are less abundant than amino acids in CR2 chondrites, with the opposite being true in CM2 and CM1/2 chondrites. We used these collective data to evaluate different primordial synthetic pathways for monoamines in carbonaceous chondrites and to understand the potential common origins these molecules may share with meteoritic amino acids.
\end{abstract}




\section{INTRODUCTION}

The study of the organic compounds from carbonaceous chondrites provides valuable insights into the available chemical inventory at the birth of the solar system, the subsequent processing that occurred inside asteroid parent bodies, and the organic chemistry preceding the origins of life on Earth (Oró, 1961; Anders, 1989; Pizzarello et al., 2006). Carbonaceous chondrites are thought to originate from water-rich asteroids and can contain up to $5 \mathrm{wt} \%$ organic carbon (Wetherill and Chapman, 1988). Among the eight groups of carbonaceous chondrites (Sears and Dodd, 1988), the moderately aqueously altered CR (Renazzo-like) and CM (Migheilike) type 2 meteorites contain the most abundant and diverse suites of water-soluble organic compounds (Cronin and Moore, 1971; Pizzarello and Cronin, 2000; Glavin et al., 2006, 2011; Martins et al., 2007; Pizzarello et al., 2008; Callahan et al., 2011).

Meteoritic amino acids have been extensively studied (see reviews and references therein: Cronin and Chang, 1993; Botta et al., 2002; Burton et al., 2012; Cobb and Pudritz, 2014; Elsila et al., 2016). Isotopic ratios, structural diversity, and enantiomeric compositions provide information about the formation mechanisms of extraterrestrial organic compounds (Epstein et al. 1987; Engel and Macko, 1997; Pizzarello and Cronin, 2000; Elsila et al., 2012). Amino acids in CR2 and CM2

carbonaceous chondrites are typically enriched in $\mathrm{D},{ }^{13} \mathrm{C}$, and ${ }^{15} \mathrm{~N}$ (Martins et al., 2007; Elsila et al., 2012), relative to their terrestrial counterparts, suggesting that the formation of these compounds or their precursor molecules occurred in cold interstellar environments (Sandford et al., 2001; Robert, 2003). The $\alpha-\mathrm{H}-\alpha$-amino acids are the most abundant amino acid species in CR2, CM2, and CM1/2 chondrites (Cronin and Pizzarello, 1983; Martins et al., 2007; Pizzarello et al., 2008; Glavin and Dworkin, 2009; Pizzarello and Holmes, 2009; Glavin et al., 2011). The enantiomeric composition of abiotically produced amino acids is racemic, in contrast to the homochirality (almost exclusively L-enantiomers) found in biological systems on Earth. A few meteoritic amino acids, most notably isovaline, have been found to exhibit an L-enantiomeric excess (L-ee; Cronin and Pizzarello, 1997; Pizzarello et al., 2003; Glavin et al., 2011; Burton et al., 2013). Isovaline is an $\alpha$-methyl- $\alpha$-amino acid that is not abundant in Earth's biosphere and is not prone to rapid racemization under aqueous conditions (Pollock et al. 1975). It has been proposed that the development of homochirality on Earth may have occurred not as a random process but instead as a consequence of the delivery of L-enantioenriched amino acids to the early Earth 
(Engel and Macko, 1997; Cronin and Pizzarello, 1997; Pizzarello et al., 2003; Glavin and Dworkin, 2009).

Aliphatic monoamines (hereafter called "amines") are organic compounds that share analogous structural backbones with amino acids; however, amines in carbonaceous chondrites have been far less studied than amino acids. Extraterrestrial methylamine and ethylamine were detected in samples collected from the coma of comet Wild 2 from the Stardust sample return mission(Sandford et al., 2006; Glavin et al., 2008), and more recently from the surface of comet 67P/Churyumov-Gerasimenko (Goesmann et al., 2015). Additionally, the abundances of various amines extracted from the CM2 Murchison meteorite and from eight Antarctic CR2 meteorites have been reported (Jungclaus et al., 1976; Pizzarello et al., 1994; Pizzarello and Holmes, 2009; Pizzarello et al., 2012). These amine detections, however, lacked isotopic and enantiomeric measurements, leaving an important gap in the understanding of the formation of meteoritic organics and the alterations these compounds may have experienced inside the parent body. Recently, we reported the compound-specific $\delta^{13} \mathrm{C}$ isotopic composition of amines in the Murchison meteorite and linked the origins of amines and amino acids to the same primordial carbon pool (Aponte et al., 2014a). The comparison of the isotopic ratios of meteoritic amines and amino acids in carbonaceous chondrites of different classes may provide important insights into the primordial origins of complex organic matter in space and to the chemical processing that happened inside the parent body (Aponte et al., 2015). Additionally, the enantiomeric compositions of chiral amines may constrain the origins of enantiomeric excesses in meteoritic amino acids, an important unanswered question for the emergence and development of Earth's homochiral life.

In this report, we investigated the molecular, isotopic, and enantiomeric compositions of amines in the Antarctic carbonaceous chondrites La Paz Icefield (LAP) 02342 (CR2), Graves Nunataks (GRA) 95229 (CR2), Lonewolf Nunataks (LON) 94101 (CM2), Lewis Cliffs (LEW) 90500 (CM2), and Allan Hills (ALH) 83100 (CM1/2), using gas chromatography coupled with mass spectrometry and isotope-ratio mass spectrometry (GC-MS/IRMS). The $\delta^{13} \mathrm{C}$ isotopic ratios of amines in these meteorites have not been previously reported; the enantiomeric composition of sec-butylamine has been previously reported but not discussed in depth (Aponte et al., 2015). Additionally, we analyzed the amino acid abundances and $\delta^{13} \mathrm{C}$ isotopic ratios in LON 94101, LEW 90500, and ALH 83100 from the same sample used for amine analysis, to directly compare 
the data on amines and amino acids while minimizing effects of potential sample heterogeneity. Our results provide information on the prebiotic synthesis of amines and amino acids and on the origins of homochirality on Earth. 


\section{MATERIALS AND METHODS}

2.1. Materials. Standards and reagents were purchased from Alfa Aesar or SigmaAldrich and used without further purification except as noted below. All glassware and sample handling tools used for the meteorite samples were rinsed with Millipore Direct Q3 UV water (18.2 $\mathrm{M} \Omega, 3$ ppb total organic carbon; hereafter referred to as "water") wrapped in aluminum foil, and then heated in a muffle furnace at $500{ }^{\circ} \mathrm{C}$ overnight. We used HPLC grade dichloromethane (DCM), semi-conductor grade $\mathrm{NaOH}$, and $6 \mathrm{M} \mathrm{HCl}$ (doubly distilled). Silica gels were bought from SiliCycle (SiliaBond ${ }^{\circledR}, 40-63 \mu \mathrm{m}$ particle size) and cleaned using methanol and DCM followed by drying under vacuum. (S)-(-)-N-(trifluoroacetyl)pyrrolidine-2-carbonyl chloride ( $S$ TPC, $97 \% e e$ ) was purchased from Sigma Aldrich. Details of the sources of specific five carbon $\left(\mathrm{C}_{5}\right)$ amino acids used as standards are available elsewhere (Glavin and Dworkin, 2009).

2.2. Meteorite samples and extraction procedure. Analyses of meteoritic amines were preformed according to previously published methods (Aponte et al., 2014a, 2015). Meteorite samples, obtained from interior chips that did not contain any visual evidence of fusion crust, were provided from the Antarctic meteorite collection at the NASA Johnson Space Center. All meteorites were individually crushed into fine powders and homogenized using a porcelain mortar and pestle in an ISO 5 positive pressure high efficiency particulate air-filtered laminar flow hood. Meteorites collected by the Antarctic Search for Meteorites (ANSMET) program are catalogued according to the geographic location where found, followed by a five digit number coding the collection year (two first digits) and specimen (three remaining digits). Additionally, in this study we show in parenthesis the parent rock number and the specific rock split for every sample. CR2 chondrites (LAP 02342, parent 0, specific 45, $411.2 \mathrm{mg}$; and GRA 95229, parent 0, specific 119, $675.1 \mathrm{mg}$ ) were analyzed for both "free" (unhydrolyzed compounds; CR2-free) and "total" (free plus hydrolyzable compounds; CR2-total) amine content. CM2 and CM1/2 chondrites (LON 94101, parent 6, specific 83, $8.0 \mathrm{~g}$ for amines, $1.9 \mathrm{~g}$ for amino acids; LEW 90500, parent 9, specific 83, $9.4 \mathrm{~g}$ for amines, $2.5 \mathrm{~g}$ for amino acids; and ALH 83100, parent 63, specific 283, 15.1 $\mathrm{g}$ for amines, $3.8 \mathrm{~g}$ for amino acids) were analyzed for total amine content (CM2-total, CM1/2total) and for total amino acid content. Portions of each powdered meteorite sample (0.4 to $1.1 \mathrm{~g})$ were flame sealed in glass ampoules with either $1 \mathrm{~mL}$ of $0.1 \mathrm{M} \mathrm{HCl}$ for the extraction of amines, or $1 \mathrm{~mL}$ of water for the extraction of amino acids, and then heated at $100{ }^{\circ} \mathrm{C}$ for $24 \mathrm{~h}$. Procedural blanks (used to quantify the concentration of amines present in the derivatization reagent prior to 
sample analyses) and a pyrolyzed serpentine (used as meteorite analog material to test the derivatization method and instrument responses) were carried through the identical extraction procedure as the meteorites.

2.3. Processing and derivatization for amine analysis. After acid-aqueous extraction, the supernatants were separated by centrifugation and the meteorite residues were rinsed three times using $0.5 \mathrm{~mL}$ of water. The extract and rinses were combined and $1 \mathrm{~mL}$ of $6 \mathrm{M} \mathrm{HCl}$ was added to each fraction. CR2 samples were divided into two equal portions for the analyses of the free and total amine content respectively; CR2-free, CR2-total, CM2-total, and CM1/2-total fractions were dried under reduced pressure. The CR2-total, CM2-total, and CM1/2-total fractions were subjected to $6 \mathrm{M} \mathrm{HCl}$ acid-vapor hydrolysis at $150{ }^{\circ} \mathrm{C}$ for $3 \mathrm{~h}$, and then dried under vacuum to remove the excess of $\mathrm{HCl}$. Next, CR2-free, CR2-total, CM2-total, and CM1/2-total dried fractions were subjected to iron ion removal by the addition of $4 \mathrm{~mL}$ of $8 \mathrm{M} \mathrm{NaOH}$. Upon precipitation of iron hydroxides, the aqueous supernatants were separated by centrifugation and the residues were rinsed twice using two portions of $4 \mathrm{~mL}$ of water. The basic extracts and water rinses were combined, readjusted to acid $\mathrm{pH}$ using $1 \mathrm{~mL}$ of $6 \mathrm{M} \mathrm{HCl}$, partially dried, consolidated for each meteorite or blank inside a $10 \mathrm{~mL}$ conical vial, and dried under vacuum. The residues were re-dissolved in $3 \mathrm{~mL}$ of $0.1 \mathrm{M} \mathrm{NaOH}$ and extracted once with $1 \mathrm{~mL}$ of DCM and twice with $0.5 \mathrm{~mL}$ of DCM. The DCM fractions $(\sim 2 \mathrm{~mL})$ were combined, passed through a plug of baked anhydrous $\mathrm{Na}_{2} \mathrm{SO}_{4}$, and rinsed once with $0.5 \mathrm{~mL}$ of DCM. The organic fractions were treated with $50 \mathrm{mg}$ of functionalized triazabicyclodecene silica gel and $50 \mathrm{uL}$ of $0.1 \mathrm{M} \mathrm{S}$-TPC. The slurry was stirred for 1 hour at room temperature, followed by the addition of $100 \mathrm{mg}$ of aminopropyl silica gel and subsequent stirring for 30 minutes at room temperature. The mixture was filtered, rinsed with $\sim 3 \mathrm{~mL}$ of DCM, blown dry with nitrogen gas and dissolved in ethyl acetate (60 to $400 \mathrm{uL})$.

2.4. Compositional and isotopic analyses. The derivatized amines were analyzed by gas chromatography coupled with mass spectrometry and isotope ratio mass spectrometry (GCMS/IRMS). The Thermo Trace GC was equipped with a $5 \mathrm{~m}$ base-deactivated fused silica guard column (Restek, $0.25 \mathrm{~mm}$ ID) and four $25 \mathrm{~m}$ length $\times 0.25 \mathrm{~mm}$ I.D. $\times 0.25 \mu \mathrm{m}$ film thickness Chirasil L-Val capillary columns (Agilent) connected with Press-Tight connectors; the GC was coupled to a Thermo DSQII electron-impact quadrupole mass spectrometer and to a Thermo MAT 253 isotope-ratio mass spectrometer via a Thermo GC-C III oxidation interface. The oven program was set as follows: initial temperature was $40{ }^{\circ} \mathrm{C}$, ramped at $12{ }^{\circ} \mathrm{C} / \mathrm{min}$ to $140{ }^{\circ} \mathrm{C}$, ramped at 4 
${ }^{\circ} \mathrm{C} / \mathrm{min}$ to $150{ }^{\circ} \mathrm{C}$ and held for $4 \mathrm{~min}$, ramped at $2{ }^{\circ} \mathrm{C} / \mathrm{min}$ to $158{ }^{\circ} \mathrm{C}$ and held for $15 \mathrm{~min}$, ramped at $20{ }^{\circ} \mathrm{C} / \mathrm{min}$ to $200{ }^{\circ} \mathrm{C}$ with a final hold of $20 \mathrm{~min}$. The carrier gas used was UHP helium (5.0 grade) at $1.4 \mathrm{~mL} / \mathrm{min}$ flow rate. The mass spectrum was used to identify and quantify compounds through comparison to reference standards and the application of a 5-point calibration curve. Targeted ion mass-to-charge ratio $(\mathrm{m} / \mathrm{z})$ was used to identify and quantify compounds as follows: $\mathrm{m} / \mathrm{z}=165.5-$ 168.5 was used for compounds $\mathbf{1}, \mathbf{2}, \mathbf{4}-\mathbf{8}, \mathbf{1 0}, \mathbf{1 1}, \mathbf{1 3}-\mathbf{1 4}, \mathbf{1 8}$ and $\mathbf{2 0}-\mathbf{2 5} ; m / z=222.5-225.5$ for compound $\mathbf{3} ; \mathrm{m} / \mathrm{z}=234.5-238.5$ for compounds $\mathbf{1 2}, \mathbf{1 5}-\mathbf{1 6}$ and $\mathbf{1 9} ; \mathrm{m} / \mathrm{z}=98.5-100.5$ for compound 9; and $m / z=112.5-114.5$ for compound 17 (see Table 1 for compound identifications). Conditions for GC-MS/IRMS analysis and measurement of $\delta^{13} \mathrm{C}$ values have been described previously (Elsila et al., 2012; Aponte et al., 2014a, 2015).

2.5. Processing and derivatization for amino acid analysis. After water extraction, the supernatants were separated by centrifugation and the meteorite residues were rinsed twice using $0.5 \mathrm{~mL}$ of water. The extract and rinses were combined, dried under reduced pressure, and subjected to $6 \mathrm{M} \mathrm{HCl}$ acid-vapor hydrolysis at $150{ }^{\circ} \mathrm{C}$ for $3 \mathrm{~h}$. The hydrolyzed fractions were dried and desalted using cation-exchange resin (AG50W-X8, 100-200 mesh, hydrogen form, Bio-Rad), and the amino acids were recovered by elution with $2 \mathrm{M} \mathrm{NH} 4 \mathrm{OH}$. After drying under vacuum, the amino acids were derivatized with $o$-phthaldialdehyde/ $N$-acetyl-L-cysteine (OPA/NAC) and immediately analyzed by positive electrospray ionization LC-FD/ToF-MS using a Waters ACQUITY ultraperformance LC and a Waters ACQUITY fluorescence detector connected in series to a Waters LCT Premier ToF-MS. The $\mathrm{C}_{5}$ amino alkanoic acids and their enantiomeric ratios in the meteorite extracts were then quantified from the peak areas generated from the exact mass chromatogram of their OPA/NAC derivatives from a minimum of 3 separate analyses. A more detailed description of the processing procedures, analytical techniques, and quantification methods for amines and amino acids is provided elsewhere (Glavin et al., 2006, 2011). 


\section{RESULTS}

3.1 Abundance and distribution of amines. Figure 1 shows GC-MS chromatograms (extracted at $m / z=165.5-168.5$ ) and typical GC-IRMS chromatograms obtained at $m / z=44$ $\left({ }^{12} \mathrm{CO}_{2}\right.$ peak) of $S$-TPC-derivatized meteoritic amines and standards. The abundances and identities of amines measured in this study are presented in Table 1. CR2 chondrites contained an overall much higher concentration of amines than CM2 and CM1/2 chondrites (Figure 2). We observed moderate variability in the concentration of amines across samples from the same petrologic type (Table 1 and Figure 2).

While investigating the free amines in the CR2 Antarctic meteorites LAP 02342 and GRA 95229, we identified $>150 \mathrm{nmol} / \mathrm{g}$ of caprolactam. Though this is the first time free caprolactam has been detected in a meteorite, it is almost certainly associated with the Nylon 6 from the bags used for the meteorites' collection; both caprolactam and Nylon 6 hydrolyze to $\varepsilon$-aminocaproic acid (EACA) (Glavin et al., 2006). Caprolactam co-eluted with methylamine, dimethylamine, ethylamine and tert-pentylamine in our GC-MS and GC-IRMS chromatograms. This made the isotopic analysis of these species impossible, though abundances (Table 1) could still be quantitated by monitoring specific ion mass-to-charge ratio $(\mathrm{m} / \mathrm{z})$ of their characteristic ions. Since EACA was detected in the CM meteorites (Table S1), we elected not to consume half of each CM sample for an unhydrolyzed study likely contaminated with caprolactam, but instead devote all the samples to maximizing the precision of the hydrolyzed analyses.

Isopropylamine was the most abundant amine in the free and hydrolyzed extracts of CR2 chondrites; however, in CM2 and CM1/2 chondrites methylamine was the most abundant amine, while the concentration of isopropylamine was similar or lower than that of ethylamine (Table 1 and Figure 2). In CR2, CM2, and CM1/2 chondrites we observed a decrease in the concentration of primary amines with increasing carbon number. No consistent trend between increasing carbon number and the abundance of secondary amines was observed (Table 1).

Among primary amines, however, we observed a tendency of amines having the amino

group $\left(-\mathrm{NH}_{2}\right)$ on a secondary carbon being more abundant than those having the $-\mathrm{NH}_{2}$ in a primary or tertiary carbon. Isopropylamine (compound 2 ; contains $-\mathrm{NH}_{2}$ on a secondary carbon) was more abundant than $n$-propylamine $\left(11\right.$; contains $-\mathrm{NH}_{2}$ on a primary carbon) in CR2 chondrites and LEW 90500 (isopropylamine and $n$-propylamine were equally abundant in LON 
94101). Additionally, the abundances of $\mathrm{C}_{4}$ and $\mathrm{C}_{5}$-primary amines normalized to the number of molecular structures possible (random distribution would assume a 33\% of each of primary, secondary, and tertiary isomers), showed that $\mathrm{C}_{4}$ primary amines having the $-\mathrm{NH}_{2}$ on a secondary carbon (compounds $\mathbf{8}$ and 10) were found in higher abundances than their isomeric compounds having the $-\mathrm{NH}_{2}$ on the primary carbon (compounds 14 and 20) or the tertiary carbon (compound 1) in all the chondrites studied here. Similarly, $\mathrm{C}_{5}$ primary amines with the $-\mathrm{NH}_{2}$ over their secondary carbon (compounds 12, 15, 16, 18 and 19), were more abundant than primary carbon (compounds $\mathbf{2 1 , 2 2}$, and $\mathbf{2 3}$ ), and tertiary carbon (compound $\mathbf{6}$ ) isomers across all samples (Table 1, Figure 3).

\subsection{Abundance, distribution and $\delta^{13} \mathrm{C}$ composition of amines in relation to their} structurally analogous amino acids. Aliphatic amines and amino acids share similar structural aliphatic backbones (Figure S2). To investigate the potential synthetic relationship between amines and amino acids through addition or loss of a carboxylic group, we compared the concentration of amines and their structurally related amino acids (Figure 4; Table S2). The amino acid content of LON 94102 (this meteorite is believed to be paired with LON 94101), LEW 90500, and ALH 83100 have been previously reported (Glavin et al., 2006; Glavin and Dworkin, 2009); however, aiming to account for sample heterogeneity in the comparison of abundances of amines and amino acids, we quantified the amino acids in these samples (Table S1; lower abundances of amino acids were found from this analysis, which may be explained by sample heterogeneity). Given the smaller amounts of LAP 02342 and GRA 95229 available for analysis, we were not able to obtain the amino acids from the same sample from which we extracted the amines; instead, we compared our amine results to previously reported amino acid abundances in the CR2 chondrites (Martins et al., 2007; Pizzarello and Holmes, 2009). With the exception of $n$-propylamine and isopropylamine in GRA 95229, we observed that amines were in lower concentrations than the sum of the concentrations of all of their structurally analogous amino acids in CR2 chondrites. In contrast, amines in CM2 and CM1/2 chondrites were more abundant than the sum of their analogous amino acids, with the exception of $n$-butylamine in LON 94102 and ALH 83100, and tert-butylamine in ALH 83100 (Figure 4; Table S2).

Table 2 shows the $\delta^{13} \mathrm{C}$ isotopic values for amines in CR2, CM2, and CM1/2 chondrites. The high molecular diversity of organic compounds in CR2 chondrites results in co-elutions of 
amines that do not allow the measurement of some amine $\delta^{13} \mathrm{C}$ isotopic values in CR2 chondrites. The $\delta^{13} \mathrm{C}$ isotopic values of amines in CR2 chondrites did not correlate with the carbon numbers of the molecules. Within the CM2 and CM1/2 chondrites, however, we observed that the ${ }^{13} \mathrm{C}$ enrichment decreases with increasing amine carbon number (Figure 5). In CR2 chondrites, methylamine and ethylamine had isotopic values ranging from -15 to $+14 \%$; in contrast, methylamine and ethylamine in CM2 and CM1/2 chondrites showed the highest $\delta^{13} \mathrm{C}$ isotopic values of any amines detected in this work, ranging from +32 to $+59 \%$ (Table 2 and Figure 5).

The $\delta^{13} \mathrm{C}$ isotopic values for some structurally related amino acids from CM2 and CM1/2 chondrites are also presented in Table $2\left(\delta^{13} \mathrm{C}\right.$ values of all amino acids are shown in Table S3). In CR2 chondrites, $\alpha$-H- $\alpha$-amino acids were mostly ${ }^{13} \mathrm{C}$-enriched relative to amines, while $\alpha$-alkyl- $\alpha$ amino acids and non- $\alpha$-amino acids were either ${ }^{13} \mathrm{C}$-depleted or within similar ${ }^{13} \mathrm{C}$ isotopic range as their structurally related amines. In CM2 and CM1/2 chondrites, the ${ }^{13} \mathrm{C}$ isotopic values of amino acids and amines do not adhere to an obvious trend.

\subsection{Enantiomeric composition of amines in the analyzed carbonaceous chondrites.}

We measured the enantiomeric ratios $(R / S)$ of three chiral amines (sec-butylamine, 3-methyl-2butylamine and sec-pentylamine) in CR2, CM2, and CM1/2 chondrites. Figure 6 shows the gas chromatogram of $(R)$ - and $(S)$-sec-butylamine (compounds 8 and 10) monitored at $m / z=165.5$ 168.5 and the mass spectra of these peaks in the analyzed extracts and standards. Comparison of the mass spectra fragmentation patterns obtained for samples and standards showed that there are no significant co-elutions or contaminants present in the analyzed samples that would affect enantiomeric quantitation. All investigated chiral amines showed enantiomeric ratios consistent with racemic compositions within experimental error (Table 3). 


\section{DISCUSSION}

4.1 Origins of meteoritic amines in CR2, CM2, and CM1/2 chondrites. The CR2 chondrites we studied (LAP 02342 and GRA 95229) have lower bulk carbon contents than the $\mathrm{CM} 2$ and $\mathrm{CM} 1 / 2$ chondrites in this work (LON 94101, LEW 90500, and ALH 83100; Alexander et al., 2007, 2012

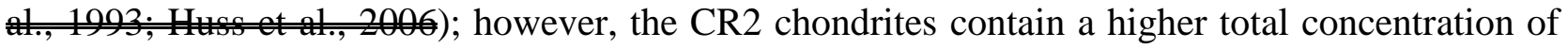
amines than the CM2 and CM1/2 chondrites (Table 1 and Figure 2). Thermometric techniques do not apply properly to CR chondrites and in general to unequilibrated chondrites (Alexander et al. 2007, 2013); thus, a more qualitative, but robust information may be gathered from the petrology, mineralogy, and insoluble organic matter of carbonaceous chondrites. Aqueous alteration is the summation of a series of complex and not fully understood processes based on the physical conditions under which molecules formed, transformed, or decomposed by the water to rock ratio, temperature, redox reactions, $\mathrm{pH}$ conditions, and time, among the main variables. In an attempt to accommodate all the complexities of the processes involved in the aqueous alteration of meteorites, Alexander et al. (2013) suggested a classification scheme based on the degree of hydration (wt.\% $\mathrm{H}$ in water and $\mathrm{OH}$ ). According to that classification, CR2 chondrites LAP 02342 and GRA 95229 were classified as type 2.5; while LON 94101, LEW 90500, Murchison and ALH 83100 were classified as 1.8, 1.6, 1.6 and 1.1 respectively (Alexander et al. 2013), were lower numbers indicate higher water content. Additionally, the ratio of the bulk water $\left(\mathrm{H}_{2} \mathrm{O}+\mathrm{OH}\right.$ wt.\%) to bulk carbon $(\mathrm{C}$ wt.\%) in these studied meteorites indicates that $\mathrm{CR} 2$ chondrites have experienced less aqueous alteration than the CM2 and CM1/2 chondrites (Alexander et al., 2012, 2013). Therefore, the different abundances of amines in CR2, CM2, and CM1/2 meteorites may be explained in part by the different levels of aqueous alteration they experienced inside their asteroid parent bodies, as higher amounts of meteoritic water have been previously linked to greater depletions of amino acids and amines inside carbonaceous chondrites (Ehrenfreund et al., 2001; Glavin et al., 2006; Aponte et al., 2015). However, CR and CM chondrites are thought to originate from two distinct asteroid parent bodies having different matrix compositions, and the concentration of ice and organics that first accreted to form those parent bodies remain unknown; thus, it may also be possible that CR2, CM2, and CM1/2 chondrites exhibit different organic compositions as a result of their distinct matrix-organic interactions (Garvie and Buseck, 2006; Alexander et al., 2013; Le Guillou and Brearley, 2014). 
Variability in the concentration of amines within meteorites of the same group and similar water content (i.e., LAP 02342 and GRA 95229 or LON 94101 and LEW 90500) has also been observed. LON 94101, LEW 90500 and ALH 83100 may derive from the same parent body; however, ALH 83100 has experienced higher aqueous alteration than LON 94101 and LEW 90500, which may explain its lower amine content. Organic variability in the CM2 and CR2 chondrites may suggest that asteroid parent bodies had heterogeneous accretions, and that processes other than aqueous alteration (e.g., impact and shock-heating) affected the abundances of amines in these chondrites' parent bodies. Further analyses of amines and other organic compounds in meteorites from the same type but with different petrologic histories will provide more information about the heterogeneity of organic compounds within parent bodies. It is worth noting, however, that the differences among samples from each carbonaceous chondrite group are smaller than the differences between these groups (Figure 2).

At least a portion of the quantified amines may have resulted from our extraction and hydrolysis methods; i.e., reaction of precursors of amines present inside the chondrite (hydrogen cyanide, formaldehyde, amides, etc.), and from the potential hydrolysis of complex insoluble macromolecules. A comparison of the amine abundances from free and total (hydrolyzed) amine extracts shown in Table 1, suggest that in most of the cases the amine abundance increase after hydrolysis. Pizzarello and Holmes (2009) previously reported the abundance of amines obtained after acid hydrolysis of hot-water extracts of the CR2 chondrites LAP 02342 and GRA 95229. Their results confirmed some of our findings (e.g., the amine content in CR2 chondrites is larger than that of CM2 and CM1/2 chondrites). Differences in the amine concentrations of those previous analyses and those reported here could be attributed to sample heterogeneity, as has been previously observed for amines and amino acids in Murchison (Cronin and Pizzarello, 1983; Krishnamurthy et al., 1992; Pizzarello et al., 2003), or to the different extraction and derivatization methodologies used, which could lead to enhancement or suppression of relative abundances in a mixture based on the polarity and steric hindrance of the amines, as we have previously discussed for Murchison (Aponte et al., 2014a).

The observed decrease in amine compound abundance with increasing carbon number is a trend previously observed for other meteoritic organic compounds such as amino acids and monocarboxylic acids, suggesting similar controls on the synthetic processes of meteoritic organics in the solar system (Cronin and Pizzarello, 1983, 1986; Huang et al., 2005; Aponte et al., 
2011). The observation that, with few exceptions, compounds with the amine on a secondary carbon are more abundant than isomers with the amine group on a primary or tertiary carbon in the $\mathrm{C}_{3}-\mathrm{C}_{5}$ amines (Figure 3, Table 1), may also reflect synthetic processes. The higher abundance of these species may suggest that meteoritic amines were formed by abiotic mechanisms that produced a complete suite of structural isomers and favoring the formation of primary amines with the $-\mathrm{NH}_{2}$ moiety on a secondary carbon of the aliphatic chains. These observations, added to the inverse relationship between abundance and molecular weight, may be important constraints for future synthetic experiments, models and theories towards the understanding of the abiotic origins of amines and other meteoritic organic compounds.

4.2 Synthetic relationship between meteoritic amines and amino acids. Amines and amino acids may have formed before the accretion of the parent body (e.g., through gas-phase reactions, in icy grains), and/or inside the asteroid mineral matrix (e.g., through aqueous, thermal processing, or impact heating); discerning between these times and locations is challenging. Our results may provide constraints for the abiotic synthesis of these compound classes inside the parent body. Multiple synthetic routes may be possible for the synthesis of meteoritic amines and amino acids (e.g., oxidation through addition of radicals); however, these compounds share aliphatic backbones and may be synthetically related through the addition or loss of $\mathrm{CO}_{2}$ (or $\mathrm{HCN}$ ), respectively (Aponte et al., 2014a, 2015). In this work we evaluated their potentially common synthetic origins based on the presence or absence of a carboxylic group on the aliphatic chain that structurally analogous amines and amino acids share (Figure S2). When comparing the concentration of individual amines and the total concentration of their structurally analogous amino acids (Figure 4; Table S2), we found that the concentration of amines was lower than that of amino acids in CR2 chondrites; in contrast, amines were more abundant than amino acids in CM2 and CM1/2 chondrites (Figure 4). The higher acidity of the $\alpha$-hydrogen in $\alpha$-H- $\alpha$-amino acids relative to that in amines may have resulted in their loss through decomposition/synthetic reactions particularly during aqueous alteration in the CM parent body (Aponte et al. 2015). Other processes such as deamination and decarboxylation of amino acids may have also been involved in the observed abundances of these organic compounds in more water-rich chondrites. Deamination of amino acids leading to carboxylic acids may be more favorable than decarboxylation leading to amines (Sato et al., 2004; Alexandrova and Jorgensen, 2011; Otake et al. 2011). Thus, the higher abundance of amines compared to amino acids in CM2 and CM1/2 chondrites may be difficult to 
explain solely by decarboxylation of amino acids, and it is instead possible that the abundance of amines and amino acids in the samples we analyzed reflect the original organic contents of their corresponding parent bodies. This observation could suggest that the amine and amino acid abundances inside CM2 and CM1/2 chondrites were originally higher than those we observed, and that aqueous processes resulted in the depletion of these organic compounds during parent body stage.

It remains possible that amines and amino acids formed through unrelated mechanisms and precursors, and that their abundances in the chondrites studied here reflect the different chemical environments where $\mathrm{CR}$ and $\mathrm{CM}$ parent bodies accreted. However, we found that the abundances of amines and amino acids share three common characteristics: 1) the abundance of both amines and amino acids are higher in less aqueously altered CR2 relative to CM2 and CM1/2 chondrites, 2) the concentrations of both compound classes decrease with increasing carbon-chain length, and 3) the concentrations of sec-butylamine and isovaline (except for amino acids in LAP 02342), are higher than those of other $\mathrm{C}_{4}$ amines and $\mathrm{C}_{5}$ amino acids, respectively. These similarities may suggest that aliphatic amines and amino acids formed from the same precursor molecules (e.g., carbonyl compounds, alcohols, nitriles), and that the different amine to amino acid ratios and molecular distributions of amines and amino acids in the studied chondrite groups are the result of the survival and processing of their precursor molecules inside the asteroid parent body.

4.3 Compound-specific carbon isotopic analyses of amines. Many organic compounds in $\mathrm{CR} 2, \mathrm{CM} 2$, and CM1/2 chondrites contain higher abundances of the heavier isotopes relative to terrestrial sources $\left(\mathrm{D} / \mathrm{H},{ }^{13} \mathrm{C} /{ }^{12} \mathrm{C},{ }^{15} \mathrm{~N} /{ }^{14} \mathrm{~N}\right)$; thus, compound-specific isotopic analyses are particularly useful to characterize the origin of meteoritic organics as extraterrestrial and to provide information about their abiotic synthetic mechanisms (Engel et al., 1990; Pizzarello et al., 1994, 2004; Elsila et al., 2012). Amines are ubiquitous in marine environments on Earth (Van Neste et al., 1987; Wang and Lee, 1990), but their $\delta^{13} \mathrm{C}$ compositions have not been reported, making it difficult to directly compare terrestrial isotopic compositions of amines with those of extraterrestrial sources. Nevertheless, the $\delta^{13} \mathrm{C}$ values of amines in CR2, CM2, and CM1/2 chondrites shown in Table 2 are ${ }^{13} \mathrm{C}$-enriched relative to terrestrial amino acids and anilines, organic compounds that are synthetically related to amines (Scott et al., 2006; Larsen et al., 2009; Elsila et al., 2011; Skarpeli-Liati et al., 2011). The ${ }^{13} \mathrm{C}$-enrichment of amines in CR2, CM2, and CM1/2 chondrites supports their formation in cold interstellar environments (Sandford et al., 2001; 
Robert, 2003), and is consistent with previous reports of other ${ }^{13} \mathrm{C}$-rich meteoritic amines and amino acids (Pizzarello et al., 2001; Huang et al., 2005; Martins et al., 2008; Aponte et al., 2014a, 2015).

Kinetically controlled chemical reactions preferentially yield ${ }^{12} \mathrm{C}$-enriched products because breaking and creating ${ }^{12} \mathrm{C}-{ }^{12} \mathrm{C}$ bonds demands slightly less energy than breaking and creating ${ }^{12} \mathrm{C}-{ }^{13} \mathrm{C}$ bonds (Park and Epstein, 1960; Abelson and Hoering, 1961; Des Marais et al. 1981). Based on these observations, Yuen et al. (1984) proposed that the systematic addition of carbon monoxide (CO) under kinetic control would result in the elongation of aliphatic chains in meteoritic organic compounds with decreasing ${ }^{13} \mathrm{C}$-content. Amines in CM2 and CM1/2 chondrites show a correlation between their ${ }^{13} \mathrm{C}$-content and molecular weight (Figure 5); in contrast, such a correlation is not observed in CR2 chondrites. The different isotopic trends observed for CR2, $\mathrm{CM} 2$ and CM1/2 aliphatic amines may suggest that a) the chain elongation mechanism proposed for amines in CM2 chondrites may have not been a preferred synthetic route for the formation of amines in CR2 chondrites, b) distinct synthetic routes were triggered from different amine precursor molecules in CR and CM parent bodies, and/or c) the higher water content in CM2 and CM1/2 chondrites resulted in the systematic destruction of amines that conserved an original lower ${ }^{13} \mathrm{C}$-content with increasing molecular weight relationship.

\subsection{Comparison of carbon isotopic compositions of amines and amino acids.} Comparisons of the carbon isotopic compositions of amines and amino acid vary widely across the sets of structurally analogous compounds in the different chondrites studied here. For example, the $\delta^{13} \mathrm{C}$ values of methylamine and ethylamine are much lower in CR2 than CM2 and CM1/2 chondrites, although their corresponding amino acids glycine and $\alpha$-alanine have similar $\delta^{13} \mathrm{C}$ values across these chondrites (Table 2). These results suggest a different formation mechanism or pool of precursors for these small, volatile amines in CR2 chondrites, and would argue against loss processes such as degassing and/or volatilization, given that such processes would have resulted in ${ }^{13} \mathrm{C}$-enriched methylamine and ethylamine through fractionation inside CR2 chondrites. Amines have a lower or similar $\delta^{13} \mathrm{C}$ value than the corresponding $\alpha$-H- $\alpha$-amino acids in $\mathrm{CR} 2$ chondrites; however, some non- $\alpha-\mathrm{H}-\alpha$-amino acids are less enriched than the corresponding amines. In CM2 and CM1/2 chondrites, the $\delta^{13} \mathrm{C}$ values of amines and both $\alpha-\mathrm{H}-\alpha$ - and non- $\alpha-\mathrm{H}-\alpha$-amino acids do not follow a clear pattern. Non- $\alpha-\mathrm{H}$ - $\alpha$-amino acids are less water soluble than their corresponding $\alpha-\mathrm{H}-\alpha$-amino acid isomers and $\mathrm{C}_{1}-\mathrm{C}_{5}$ amines, and the $\alpha-\mathrm{H}$ in $\alpha-\mathrm{H}-\alpha$-amino acids is more acidic 
than that of amines; therefore, it is reasonable to expect that $\alpha-\mathrm{H}-\alpha$-amino acids would be ${ }^{13} \mathrm{C}$ depleted relative to non- $\alpha-\mathrm{H}-\alpha$-amino acids and their structurally analogous amines as a result of isotopic fractionation from aqueous processes inside the parent body. With this current data set of isotopic values, however, it remains difficult to assess both the synthetic relationship between meteoritic amines and amino acids, if any, and the role of aqueous processes in the $\delta^{13} \mathrm{C}$ fractionation of meteoritic amines.

4.5 Racemic composition of chiral amines and isovaline. The enantiomeric composition of organic compounds in meteorites has been a question of great interest since the discovery of Lenantiomeric excesses in certain amino acids and the suggestion that the delivery of these enantiomerically enriched compounds to the early Earth may have contributed to the origin of Earth's homochiral life (Engel and Macko, 1997; Pizzarello et al., 2003; Cronin and Pizzarello, 1997; Glavin and Dworkin, 2009; Glavin et al., 2011, 2012; Burton et al., 2013). If the aliphatic portion of meteoritic amines and amino acids formed through common synthetic mechanisms, then comparing their enantiomeric compositions may aid in understanding these synthetic pathways and the effects of parent body processes on enantioenrichment.

The measurements reported here of three chiral amines (sec-butylamine, sec-pentylamine, and 2-methylbutylamine; Table 3) represent one of the few analyses of enantiomeric compositions for compounds other than amino acids in carbonaceous chondrites. The racemic distributions of amines in the samples analyzed here, are consistent with the racemic compositions of analogous aliphatic monocarboxylic acids found in meteorites from various petrologic types (Aponte et al., 2014b) and with the measurements of racemic amines in the Murchison and Orgueil meteorites (Aponte et al. 2014a; Aponte et al. 2015). Additionally, our results are supported by anisotropy studies suggesting that ultra-violet circularly polarized light (UVCPL) may induce enantiomeric excess aliphatic amino acids, but not in amines and carboxylic acids (Meinert et al., 2011, 2016, Myrgorodska et al., 2016).

While this work was under review for publication, Pizzarello and Yarnes (2016) reported enantiomeric excesses of $(S)$-sec-butylamine and $(S)$-sec-pentylamine ranging from 0 to $66 \%$ in the CR2 chondrites LAP 02342 and GRA 95229. The authors of that work attributed the differences in the enantiomeric enrichments they found relative to those presented here and elsewhere (Aponte et al. 2015) to sample heterogeneity. Although heterogeneity is always a potential concern when 
comparing analyses of meteoritic samples, a more parsimonious explanation may exist in in the methodology used in that report.

The amino acid isovaline was also found to be racemic in LON 94101 and LEW 90500 (these results are in agreement with those by Galvin and Dworkin (2009), who reported nearly racemic L-isovaline from LON 94012 and LEW 90500), however, a non-racemic composition of isovaline was found in ALH 83100; for the CR2 chondrites, an L-isovaline enantiomeric excess of 3\% was previously reported for a separate portion of GRA 95229 and racemic isovaline was reported in LAP 02342 (Pizzarello et al. 2008; Pizzarello and Holmes, 2009). Previous measurements of L-excesses of isovaline and other amino acids in meteorites have been attributed to amplification of a small initial excess, perhaps through parent body processes driven by aqueous alteration (Viedma, 2001; Klussman et al., 2006; Glavin et al., 2012); this may have occurred in GRA 95229 and ALH 83100. However, the racemic measurements of the amines in all meteorites studied in this work and of isovaline in LON 94101, LEW 90500, and LAP 02342 suggest that the amines and isovaline in these meteorites either were not subjected to any initial symmetrybreaking event, or that there was not an amplification process sufficient to raise any enantiomeric excess present above detection limits. 


\section{CONCLUSION}

We have analyzed the molecular distributions, compound-specific $\delta^{13} \mathrm{C}$ isotopic values and enantiomeric compositions of amines from the acid-water extracts of CR2, CM2, and CM1/2 chondrites, and have considered this data in relation to potential synthetic routes for their formation and in relation to their structurally analogous amino acids:

- We observed higher abundances of amines in CR2 chondrites compared to CM2 and CM1/2 chondrites. Amine distributions differ between the two carbonaceous chondrite groups, with isopropylamine being the most abundant amine species in CR2 chondrites, while methylamine is the most abundant amine species in CM2 and CM1/2 chondrites. In addition, we found a decrease in amine concentration with increasing carbon number, and that compounds with the amino moiety on a secondary carbon tended to be more abundant than isomers with the amino group on a primary or tertiary carbon.

- We did not observe a correlation between the number of carbon atoms and the ${ }^{13} \mathrm{C}$-content of amines in CR2 chondrites; however, in CM2 and CM1/2 chondrites, the ${ }^{13} \mathrm{C}$ content of amines decreased with increasing carbon number. These observations may suggest that the systematic addition of carbon monoxide (CO) under kinetic control previously proposed was not a universal synthetic route for the synthesis of meteoritic organic compounds, and/or that the relationship found for $\delta^{13} \mathrm{C}$ isotopic ratios and chain length in amines from $\mathrm{CM} 2$ and $\mathrm{CM} 1 / 2$ chondrites resulted from the selective destruction of ${ }^{12} \mathrm{C}$-rich molecules through aqueous processes.

- We found that amino acids are more abundant than amines in CR2 chondrites, while the reverse is true in $\mathrm{CM} 2$ and $\mathrm{CM} 1 / 2$ chondrites. These results may be either original signatures of distinct parent bodies or may suggest that aqueous processes destructive towards amino acids were active on the CM parent body.

- We found racemic compositions of sec-butylamine across the CR2 and CM2 and CM1/2 chondrites we investigated, and racemic isovaline in three of the five meteorites. These results suggest that the amines may have originated from racemic precursors or that the enantiomeric amplification mechanisms previously proposed for the L-enantioenrichment of meteoritic amino acids did not take place in these samples. 
Future experimental analyses and theoretical modeling of amines and other meteoritic organic species in carbonaceous chondrites from different petrologic types may further our understanding about the origins of the basic building blocks of life on Earth. 


\section{ACKNOWLEDGEMENTS}

US Antarctic meteorite samples are recovered by the Antarctic Search for Meteorites (ANSMET) program which has been funded by NSF and NASA, and characterized and curated by the Department of Mineral Sciences of the Smithsonian Institution and Astromaterials Curation Office at NASA Johnson Space Center. J.C.A. acknowledges support from the NASA Postdoctoral Program at the Goddard Space Flight Center, administered by Oak Ridge Associated Universities and the NASA Astrobiology Institute through a contract with NASA. This research was supported by the NASA Astrobiology Institute and the Goddard Center for Astrobiology, and a grant from the Simons Foundation (SCOL award 302497 to J.P.D.). The authors acknowledge Associate Editor Eric Quirico and two anonymous reviewers for their helpful comments on this manuscript. 


\section{REFERENCES}

Abelson P. H. and Hoering T. C. (1961) Carbon isotope fractionation in formation of amino acids by photosynthetic organisms. Proc. Natl. Acad. Sci. USA 47, 623-632.

Alexander C. M. O’D., Fogel M. L., Yabuta H. and Cody G. D. (2007) The origin and evolution of chondrites recorded in the elemental and isotopic compositions of their macromolecular organic matter. Geochim. Cosmochim. Acta 71, 4380-4403.

Alexander C. M. O. D., Newsome S. D., Fogel M. L., and Cody G. D. (2010) Deuterium enrichments in chondritic macromolecular material: Implications for the origin and evolution of water, organics, and asteroids. Geochim. Cosmochim. Acta 74, 4417-4437.

Alexander C. M. O’D., Bowden R., Fogel M. L., Howard K. T., Herd C. D. K. and Nittler L. R. (2012) The provenances of asteroids, and their contributions to the volatile inventories of the terrestrial planets. Science 337, 721-723.

Alexander C. M. O’D., Howard K., Bowden R. and Fogel M. L. (2013) The classification of CM and $\mathrm{CR}$ chondrites using bulk $\mathrm{H}, \mathrm{C}$ and $\mathrm{N}$ abundances and isotopic compositions. Geochim. Cosmochim. Acta 123, 244-260.

Alexandrova A. N., and Jorgensen W. L. (2011) On the mechanism and rate of spontaneous decomposition of amino acids. J. Phys. Chem. B 115, 13624-13632.

Anders E. (1989) Pre-biotic organic matter from comets and asteroids. Nature 342, 255-257.

Aponte J. C., Alexandre M. R., Wang Y., Brearley A. J., Alexander C. O'D. and Huang Y. (2011) Effects of secondary alteration on the composition of free and IOM-derived monocarboxylic acids in carbonaceous chondrites. Geochim. Cosmochim. Acta 75, 23092323.

Aponte J. C., Dworkin J. P., and Elsila J. E. (2014a) Assessing the origins of aliphatic amines in the Murchison meteorite from their compound-specific carbon isotopic ratios and enantiomeric composition. Geochim. Cosmochim. Acta 141, 331-345.

Aponte J. C., Tarozo R., Alexandre M. R., Alexander C. M. O’D., Charnley S. B., Hallmann C., Summons R. and Huang Y. (2014b) Chirality of meteoritic free and IOM-derived monocarboxylic acids and implications for prebiotic organic synthesis. Geochim. Cosmochim. Acta 131, 1-12.

Aponte J. C., Dworkin J. P., and Elsila J. E. (2015) Indigenous aliphatic amines in the aqueously altered Orgueil meteorite. Meteorit. Planet. Sci. 50, 1733-1749.

Bell M. B., Avery L. W., MacLeod J. M., and Matthews H. E. (1992) The excitation temperature of $\mathrm{HC}_{9} \mathrm{~N}$ in the circumstellar envelope. Astrophys. J. 400, 551-555.

Botta O., Glavin D. P., Kminek G., and Bada J. L. (2002) Relative amino acid concentrations as a signature for parent body processes of carbonaceous chondrites. Orig. Life Evol. Biosph. 32, 143-163.

Burton A. S., Stern J. C., Elsila J. E., Glavin D. P. and Dworkin J. P. (2012) Chem. Soc. Rev. 41, 5459-5472. 
Burton A. S., Elsila J. E., Hein J. E., Glavin D. P. and Dworkin J. P. (2013) Extraterrestrial amino acids identified in metal-rich $\mathrm{CH}$ and $\mathrm{CB}$ carbonaceous chondrites from Antarctica. Meteorit. Planet. Sci. 48, 390-402.

Callahan M .P., Smith K. E., Cleaves II H. J., Ruzicka J., Stern J. C., Glavin D. P., House C. H. and Dworkin J. P. (2011) Carbonaceous meteorites contain a wide range of extraterrestrial nucleobases. Proc. Natl. Acad. Sci. U.S.A. 108, 13995-13998.

Charnley S. B., Ehrenfreund P., Millar T. J., Boogert A. C. A., Markwick A. J., Butner H. M., Ruiterkamp R. and Rodgers S. D. (2004) Observational tests for grain chemistry: posterior isotopic labelling. Monthly Not. Royal Astron. Soc. 347, 157-162.

Clayton R. N. and Mayeda T. K. (1999) Oxygen isotope studies of carbonaceous chondrites. Geochim. Cosmochim. Acta 63, 2089-2104.

Cobb A. K. and Pudritz R. E. (2014) Nature's starships. I. Observed abundances and relative frequencies of amino acids in meteorites. Astrophys. J. 783, 140 (12pp).

Cronin J. R. and Moore C. B. (1971) Amino acid analyses of the Murchison, Murray, and Allende carbonaceous chondrites. Science 172, 1327-1329.

Cronin J. R. and Pizzarello S. (1983) Amino acids in meteorites. Adv. Space Res. 3, 5-18.

Cronin J. R. and Pizzarello S. (1986) Amino acids of the Murchison meteorite. III. Seven carbon acyclic primary $\alpha$-amino alkanoic acids. Geochim. Cosmochim. Acta 50, 2419-2427.

Cronin J. R. and Chang S. (1993) Organic matter in meteorites: molecular and isotopic analyses of the Murchison meteorite. In The Chemistry of Life's Origins (eds. J. M. Greenberg, C. X. Mendoza-Gómez and V. Pirronello), pp. 209-258. Kluwer Academic Publishers.

Cronin J. R. and Pizzarello S. (1997) Enantiomeric excesses in meteoritic amino acids. Science 275, 951-955.

Des Marais D. J., Donchin J. H., Nehring N. L. and Truesdell A. H. (1981) Molecular carbon isotopic evidence for the origin of geothermal hydrocarbons. Nature 292, 826828.Ehrenfreund P., Glavin D. P., Botta O., Cooper G. and Bada J. L. (2001) Extraterrestrial amino acids in Orgueil and Ivuna: Tracing the parent body of CI type carbonaceous chondrites. Proc. Natl. Acad. Sci. U.S.A. 98, 2138-2141.

Elsila J. E., Callahan M. P., Glavin D. P., Dworkin J. P. and Buckner H. (2011) Distribution and stable isotopic composition of amino acids from fungal peptaibiotics: Assessing the potential for meteoritic contamination. Astrobiology 11, 123-133.

Elsila J.E., Charnley S.B., Burton A.S., Glavin D.P. and Dworkin J.P. (2012) Compound-specific carbon, nitrogen, and hydrogen isotopic ratios for amino acids in CM and CR chondrites and their use in evaluating potential formation pathways. Meteor. Planet. Sci. 47, 15171536.

Elsila J. E., Aponte J. C., Blackmond D. G., Burton A. S., Dworkin J. P. and Glavin D. P. (2016) Meteoritic amino acids: Diversity in compositions reflects parent body histories. ACS Cent. Sci. DOI: 10.1021/acscentsci.6b00074.

Engel M. H., Macko S. A. and Silfer J. A. (1990) Carbon isotope composition of individual amino acids in the Murchison meteorite. Nature 348, 47-49. 
Engel M. H. and Macko S. A. (1997) Isotopic evidence for extraterrestrial non-racemic amino acids in the Murchison meteorite. Nature 389, 265-268.

Epstein S., Krishnamurthy R. V., Cronin J. R., Pizzarello S. and Yuen G. U. (1987) Unusual stable isotope ratios in amino-acid and carboxylic-acid extracts from the Murchison meteorite. Nature 326, 477-479.

Garvie L. A. J. and Buseck P. R. (2006) Carbonaceous materials in the acid residue from the Orgueil carbonaceous chondrite meteorite. Meteorit. Planet. Sci. 41, 633-642.

Glavin D. P., Dworkin J. P., Aubrey A., Botta O., Doty III J. H., Martins Z. and Bada J. L. (2006) Amino acid analyses of Antarctic CM2 meteorites using liquid chromatography-time of flight-mass spectrometry. Meteor. Planet. Sci. 41, 889-902.

Glavin D. P., Dworkin J. P. and Sandford S. A. (2008) Detection of cometary amines in samples returned by Stardust. Meteorit. Planet. Sci. 43, 399-413.

Glavin D. P. and Dworkin J. P. (2009) Enrichment of the amino acid L-isovaline by aqueous alteration on CI and CM meteorite parent bodies. Proc. Natl. Acad. Sci. USA 106, 54875492.

Glavin D. P., Callahan M. P., Dworkin J. P., and Elsila J. E. (2011) The effects of parent body processes on amino acids in carbonaceous chondrites. Meteorit. Planet. Sci. 45, 1948-1972.

Glavin D. P., Elsila J. E., Burton A. S., Callahan M. P., Dworkin J. P., Hilts R. W., and Herd C. D. K. (2012) Unusual nonterrestrial L-proteinogenic amino acid excesses in the Tagish Lake meteorite. Meteorit. Planet. Sci. 47, 1348-1364.

Goesmann F., Rosenbauer H., Bredehöft J. H., Cabane M., Ehrenfreund P., Gautier T., Giri C., Krüger H., Le Roy L., MacDermott A. J., McKenna-Lawlor S., Meierhenrich U. J., Muñoz-Caro G. M., Raulin F., Roll R., Steele A., Steininger H., Sternberg R., Szopa C., Thiemann W. and Ulamec S. (2015) Organic compounds on comet 67P/ChuryumovGerasimenko revealed by COSAC mass spectrometry. Science 349, aab0689.

Howard K. T., Alexander C. M. O’D., Schrader D.L., Dyl K. A. 2015. Classification of hydrous meteorites (CR, $\mathrm{CM}$ and $\mathrm{C} 2$ ungrouped) by phyllosilicate fraction: PSD-XRD modal mineralogy and planetesimal environments. Geochim. Cosmochim. Acta 149, 206-222.

Huang Y., Wang Y., Alexandre M. R., Lee T., Rose-Petruck C., Fuller M. and Pizzarello S. (2005) Molecular and compound-specific isotopic characterization of monocarboxylic acids in carbonaceous meteorites. Geochim. Cosmochim. Acta 69, 1073-1084.

Hudson R. L., Lewis A. S., Moore M. H., Dworkin J. P. and Martin M. P. (2009) Enigmatic isovaline: Investigating the stability, racemization, and formation of a non-biological meteoritic amino acid. ASP Conf. Ser. 420, 157-162.

Hus. G. R., Rubin A. E. and Grosman J. N. (2006)-Thermalmomism in

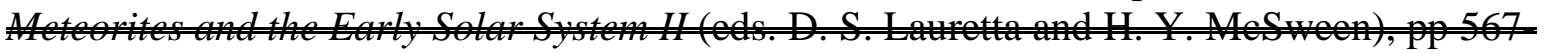
586. University af Arizona Press: Tues, AZ.

Jungclaus G. A., Cronin J. R., Moore C. B. and Yuen G. U. (1976) Aliphatic amines in the Murchison meteorite. Nature 261, 126-128. 
Klussman M., Iwamura H., Mathew S. P., Wells D. H., Jr., Pandya U., Armstrong A., and Blackmond D. G. (2006) Thermodynamic control of asymmetric amplification in amino acid catalysis. Nature 441, 621-623.

Krishnamurthy R.V., Epstein S., Cronin J.R., Pizzarello S. and Yuen G.U. (1992) Isotopic and molecular analyses of hydrocarbons and monocarboxylic acids of the Murchison meteorite. Geochim. Cosmochim. Acta 56, 4045-4058.

Larsen T., Taylor D. L., Leigh M. B. and O’Brien D. M. (2009) Stable isotope fingerprinting: a novel method for identifying plant, fungal, or bacterial origins of amino acids. Ecology 90, 3526-3535.

Le Guillou C. and Brearley A. J. (2014) Relationships between organics, water and early stages of aqueous alteration in the pristine CR3.0 chondrite MET 00426. Geochim. Cosmochim. Acta 131, 344-367.

Lerner N. R., Peterson E. and Chang S. (1993) The Strecker synthesis as a source of amino acids in carbonaceous chondrites-Deuterium retention during synthesis. Geochim. Cosmochim. Acta 57, 4713-4723.

Martins Z., Alexander C. M. O. D., Orzechowska G. E., Fogel M. L. and Ehrenfreund P. (2007) Indigenous amino acids in primitive CR meteorites. Meteorit. Planet. Sci. 42, 2125-2136.

Martins Z., Botta O., Fogel M. L., Sephton M. A., Glavin D. P., Watson J. S., Dworkin J. P., Schwartz A. W. and Ehrenfreund P. (2008) Extraterrestrial nucleobases in the Murchison meteorite. Earth and Planetary Science Letters 270, 130-136.

Meinert C., Bredehöft J. H., Filippi J. J., Baraud Y., Nahon L., Wien F., Jones N. C., Hoffmann S. V., and Meierhenrich U. J. (2012) Anisotropy spectra of amino acids. Angew. Chem., Int. Ed. 51, 4484-4487.

Meinert C., Jones N. C., Hoffmann S. V., Meierhenrich U. J. (2016) Anisotropy spectroscopy of chiral alcohols, amines, and monocarboxylic acids: Implications for the analyses of extraterrestrial samples. J. Photochem. Photobiol., A DOI: 10.1016/j.jphotochem.2015.12.018.

Myrgorodska I., Meinert C., Hoffmann S. V., Jones N. C., Nahon L., and Meierhenrich U. J. (2016) Light on chirality: Absolute asymmetric formation of chiral molecules relevant in prebiotic evolution. ChemPlusChem, DOI: 10.1002/cplu.201600214.

Öberg K. I., Guzman V. V., Furuya K., Qi C., Aikawa Y., Andrews S. M., Loomis R., and Wilner D. J. (2015) The comet-like composition of a protoplanetary disk as revealed by complex cyanides. Nature 520, 198-201.

Oró, J. (1961) Comets and the formation of biochemical compounds on the primitive Earth. Nature 190, 389-390.

Otake T., Taniguchi T., Furukawa Y., Kawamura F., Nakazawa H., and Kakegawa T. (2001) Stability of amino acids and their oligomerization under high-pressure conditions: Implications for prebiotic chemistry. Astrobiology 11, 799-813.

Park R., S. Epstein. 1960. Carbon isotope fractionation during photosynthesis. Geochim. Cosmochim. Acta 21, 110-126. 
Peltzer E. T. and Bada J. L. (1978) Alpha-hydroxycarboxylic acids in the Murchison meteorite Nature 272, 443-444.

Peltzer E. T., Bada J. L., Schlesinger G. and Miller S. L. (1984) The chemical conditions on the parent body of the Murchison meteorite: some conclusions based on amino, hydroxy and dicarboxylic acids. Adv. Space Res. 4, 69-74.

Pizzarello S., Feng X., Epstein S. and Cronin J. R. (1994) Isotopic analyses of nitrogenous compounds from the Murchison meteorite: ammonia, amines, amino acids, and polar hydrocarbons. Geochim. Cosmochim. Acta 58, 5579-5587.

Pizzarello S., and Cronin J. R. (2000) Non-racemic amino acids in the Murray and Murchison meteorites. Geochim. Cosmochim. Acta 64, 329-338.

Pizzarello S., Huang Y. S., Becker L., Poreda R. J., Nieman R. A., Cooper G. and M. Williams (2001) The organic content of the Tagish Lake meteorite. Science 293, 2236-2239.

Pizzarello S. (2002) The chiral amines of the Murchison meteorite: A preliminary characterization (abstract). In Lunar and Planetary Science XXXIII, Abstract \#1233. Lunar and Planetary Institute, Houston.

Pizzarello S., Zolensky M. and Turk K. A. (2003) Non racemic isovaline in the Murchison meteorite: chiral distribution and mineral association. Geochim. Cosmochim. Acta 67, 1589-1595.

Pizzarello S., Huang Y. and Fuller M. (2004) The carbon isotopic distribution of Murchison amino acids. Geochim. Cosmochim. Acta 68, 4963-4969.

Pizzarello S., Cooper G. W. and Flynn G. J. (2006) The nature and distribution of the organic material in carbonaceous chondrites and interplanetary dust particles. In Meteorites and the Early Solar System II (eds. D. S. Lauretta and H. Y. McSween), pp 625-651. University of Arizona Press: Tucson, AZ.

Pizzarello S., Huang Y. and Alexandre M. R. (2008) Molecular asymmetry in extraterrestrial chemistry: Insights from a pristine meteorite. Proc. Natl. Acad. Sci. USA 105, 3700-3704.

Pizzarello S. and Holmes W. (2009) Nitrogen-containing compounds in two CR2 meteorites: ${ }^{15} \mathrm{~N}$ composition, molecular distribution and precursor molecules. Geochim. Cosmochim. Acta $73,2150-2162$.

Pizzarello S., Williams L. B., Lehman J., Holland G. P. and Yarger J. L. (2011) Abundant ammonia in primitive asteroids and the case for a possible exobiology. Proc. Natl. Acad. Sci. USA 108, 4303-4306.

Pizzarello S., Schrader D. L., Monroe A. A. and Lauretta D. S. (2012) Large enantiomeric excesses in primitive meteorites and the diverse effects of water in cosmochemical evolution. Proc. Natl. Acad. Sci. USA 109, 11949-11954.

Pizzarello S. (2014) The nitrogen isotopic composition of meteoritic HCN. Astroph. J. Lett. 796, L25 (4pp).

Pizzarello S. and Yarnes T. (2016) Enantiomeric excesses of chiral amines in ammonia-rich carbonaceous meteorites. Earth Planet. Sci. Lett. 443, 176-184. 
Pollock G. E., Cheng C.-N., Cronin S. E. and Kvenvolden K. A. (1975) Stereoisomers of isovaline in the Murchison meteorite. Geochim. Cosmochim. Acta 39, 1571-1573.

Robert F. (2003) The D/H ratio in chondrites. Space Sci. Rev. 106, 87-101.

Sandford S. A., Bernstein M. P. and Dwokin J. P. (2001) Assessment of the interstellar processes leading to deuterium enrichment in meteoritic organics. Meteor. Planet. Sci. 36, 11171133.

Sandford S. A., Aléon J., Alexander C. M. O’D., Araki T., Bajt S., Baratta G. A., Borg J., Brucato J. R., Burchell M. J., Busemann H., Butterworth A., Clemett S. J., Cody G., Colangeli L., Cooper G., D’Hendecourt L., Djouadi Z., Dworkin J. P., Ferrini G., Fleckenstein H., Flynn G. J., Franchi I. A., Fries M., Gilles M. K., Glavin D. P., Gounelle M., Grossemy F., Jacobsen C., Keller L. P., Kilcoyne D., Leitner J., Matrajt G., Meibom A., Mennella V., Mostefaoui S., Nittler L. R., Palumbo M. E., Robert F., Rotundi A., Snead C. J., Spender M. K., Steele A., Stephan T., Tyliszczak T., Westphal A. J., Wirick S., Wopenka B., Yabuta H., Zare R. N. and Zolensky M. (2006) Organics captured from comet Wild 2 by the Stardust spacecraft. Science 314, 1720-1724.

Sato N., Quitain A. T., Kang K., Daimon H., and Fujie K. (2004) Reaction kinetics of amino acid decomposition in high-temperature and high-pressure water. Ind. Eng. Chem. Res. 43, 3217-3222.

Scott J. H., O’Brien D. M., Emerson D., Sun H., McDonald G. D., Salgado A. and Fogel M. L. (2006) An examination of the carbon isotope effects associated with amino acid biosynthesis. Astrobiology 6, 867-880.

Sears D. W. G. and Dodd R. T. (1988). Overview and classification of meteorites. In Meteorites and the Early Solar System (ed. J. F. Kerridge and M. S. Matthews), pp. 3-31. University of Arizona Press: Tucson, AZ.

Skarpeli-Liati M., Turgeon A., Garr A. N., Arnold W. A., Cramer C. J. and Hofstetter T. B. (2011) $\mathrm{pH}$-Dependent equilibrium isotope fractionation associated with the compound specific nitrogen and carbon isotope analysis of substituted anilines by SPME-GC/IRMS. Anal. Chem. 83, 1641-1648.

Theule P., Borget F., Mispelaer F., Danger G., Duvernay F., Guillemin J. C. and Chiavassa T. (2011) Hydrogenation of solid hydrogen cyanide $\mathrm{HCN}$ and methanimine $\mathrm{CH}_{2} \mathrm{NH}$ at low temperature. Astron. Astrophysics. 534, A64 (6pp).

Tielens A. G. G. M. and Hagen W. (1982) Model calcualtions of the molecular composition of interstellar grains mantles. Astron. Astrophysics. 114, 245-260.

Van Neste A., Duce R. A. and Lee C. (1987) Methylamines in the marine atmosphere. Geophys. Res. Lett. 14, 711-714.

Viedma C. (2001) Enantiomeric crystallization from DL-aspartic and DL-glutamic acids: Implications for biomolecular chirality in the origin of life. Orig. Life Evol. Biosph. 31, 501-509.

Wang X. C. and Lee C. (1990) The distribution and adsorption behavior of aliphatic amines in marine and lacustrine sediments. Geochim. Cosmochim. Acta 54, 2759-2774. 
Wetherill G. W. and Chapman C. R. (1988) Asteroids and meteorites. In Meteorites and the Early Solar System (ed. J. F. Kerridge and M. S. Matthews), pp. 35-67. University of Arizona Press: Tucson, AZ.

Yuen, G., Blair, N., Des Marais, D.J. and Chang S. (1984) Carbon isotopic composition of individual, low molecular weight hydrocarbons and monocarboxylic acids from the Murchison meteorite. Nature 307, 252-254.

Zolensky M., Baret R., and Browning $\mathrm{L}$. (1993) Mineralogy and composition of matrix and ehondule rims in a a 57,3123-31448. 
Figure 1. Panels A.1-A.9 show positive electron impact GC-MS chromatograms of $S$-TPC-derivatized acid-vapor hydrolyzed amines in CR2, CM2, and CM1/2 meteorites, $S$-TPC-derivatized acid-vapor-hydrolyzed procedural blanks and $S$-TPC-derivatized standards. Panels B.1-B.2 shows typical GC-IRMS chromatograms obtained at $\mathrm{m} / z=44\left({ }^{12} \mathrm{CO}_{2}\right.$ peak $)$ during carbon compound-specific isotope analysis of $S$-TPC-derivatized standards and acid-vapor-hydrolyzed amines. The identities of the peaks and structures are presented in Table 1 and Figure S1 respectively. Unidentified peaks represent compounds that could not be adequately identified or analyzed because of co-elutions or insufficient sensitivity. P: phthalate, R: reagent ( $S$-TPC acid), U: Unknown compound.
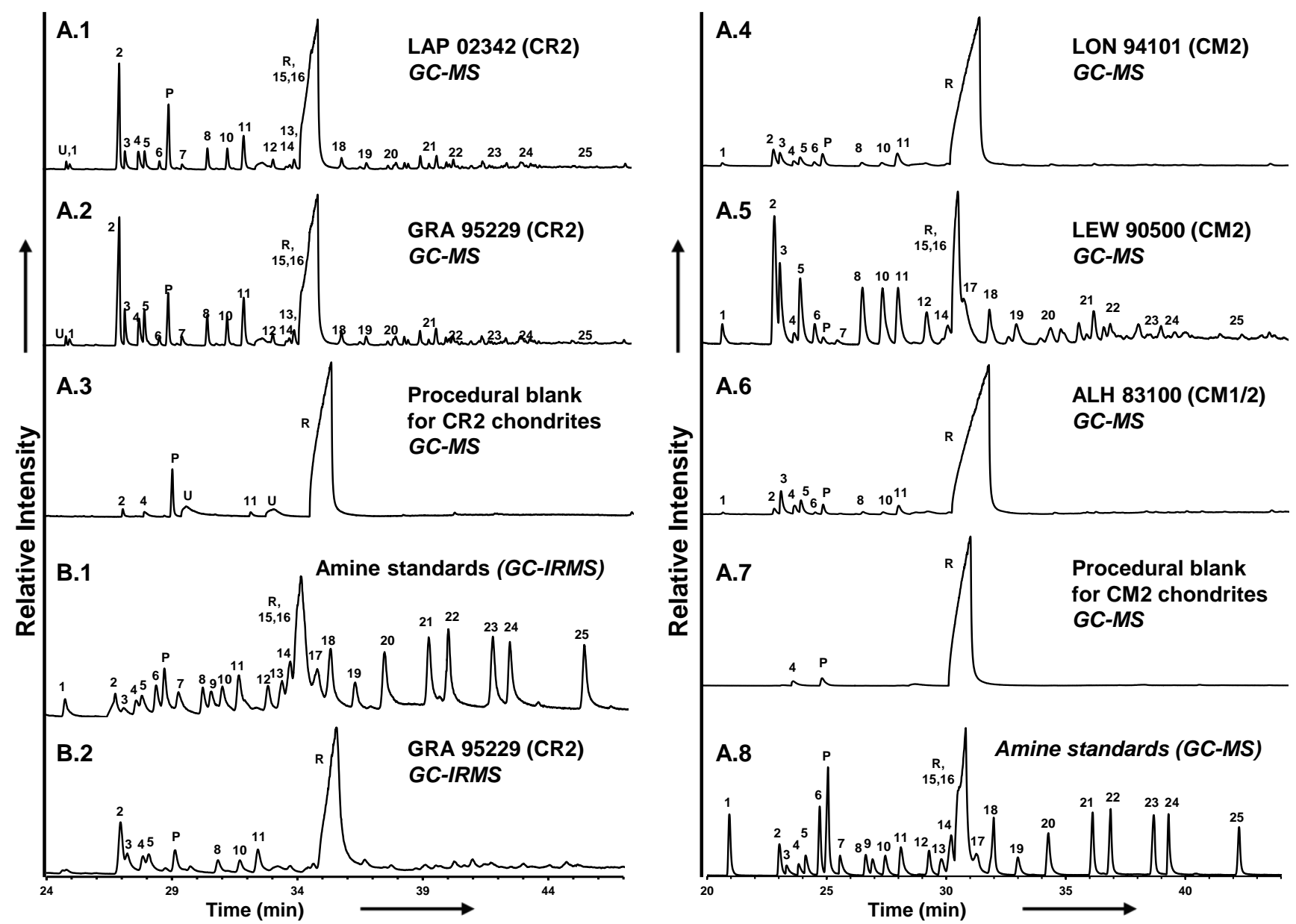
Figure 2. Abundances of $\mathrm{C}_{1}-\mathrm{C}_{4}$ amines in acid-hydrolyzed hot water extracts from $\mathrm{CR} 2, \mathrm{CM} 2$, and $\mathrm{CM} 1 / 2$ carbonaceous chondrites. Meteorites are arranged in order of increasing aqueous alteration.

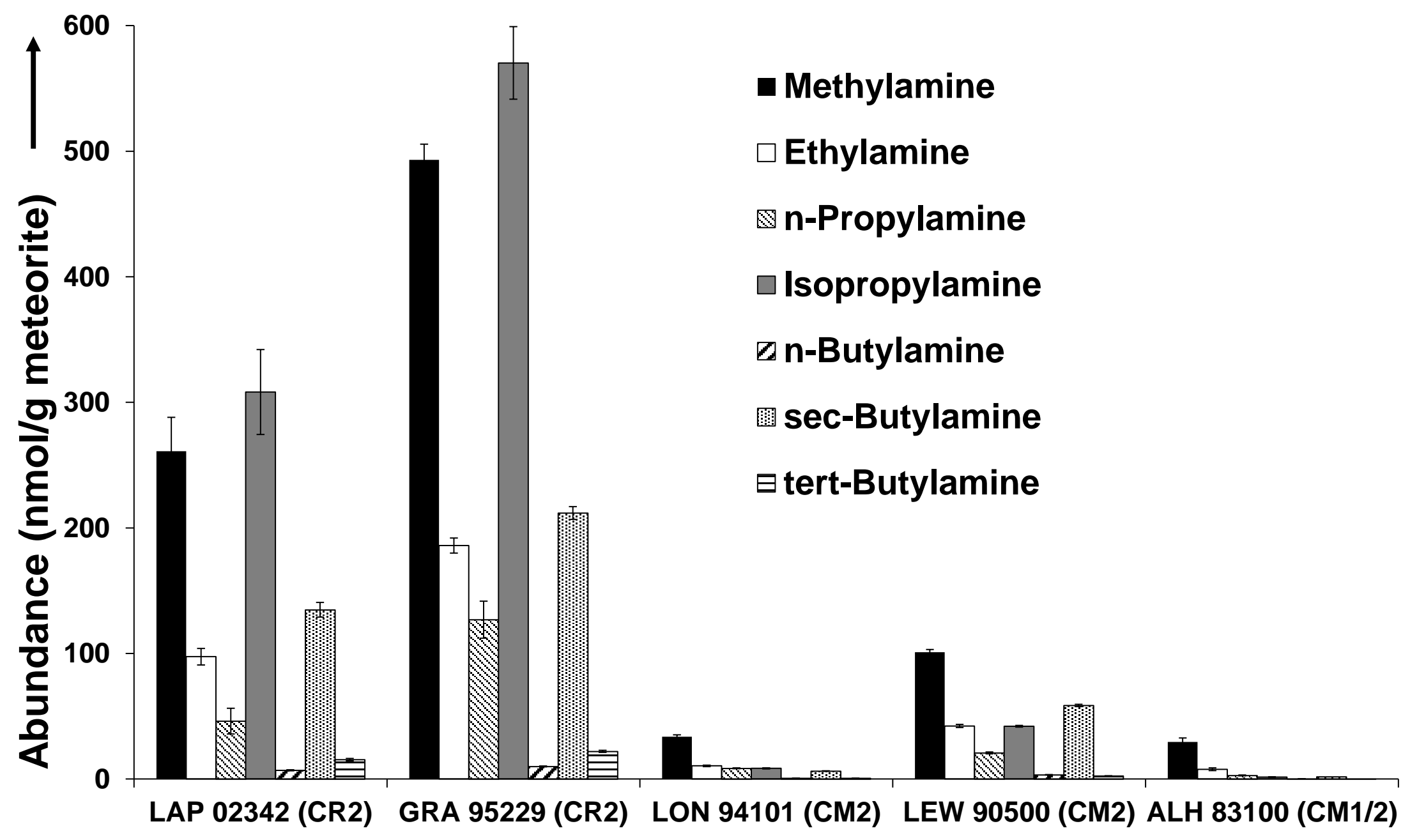


Figure 3. The abundances of $\mathrm{C}_{4}$ - and $\mathrm{C}_{5}$-primary amines having the $-\mathrm{NH}_{2}$ moiety in a secondary carbon normalized to the number of molecular structures are larger than those of their corresponding primary-carbon and tertiary-carbon isomers in CR2, CM2, and CM1/2 chondrites. A random distribution during formation would create $33 \%$ each of the three isomeric groups. Compound number from Table 1 is shown in parentheses.

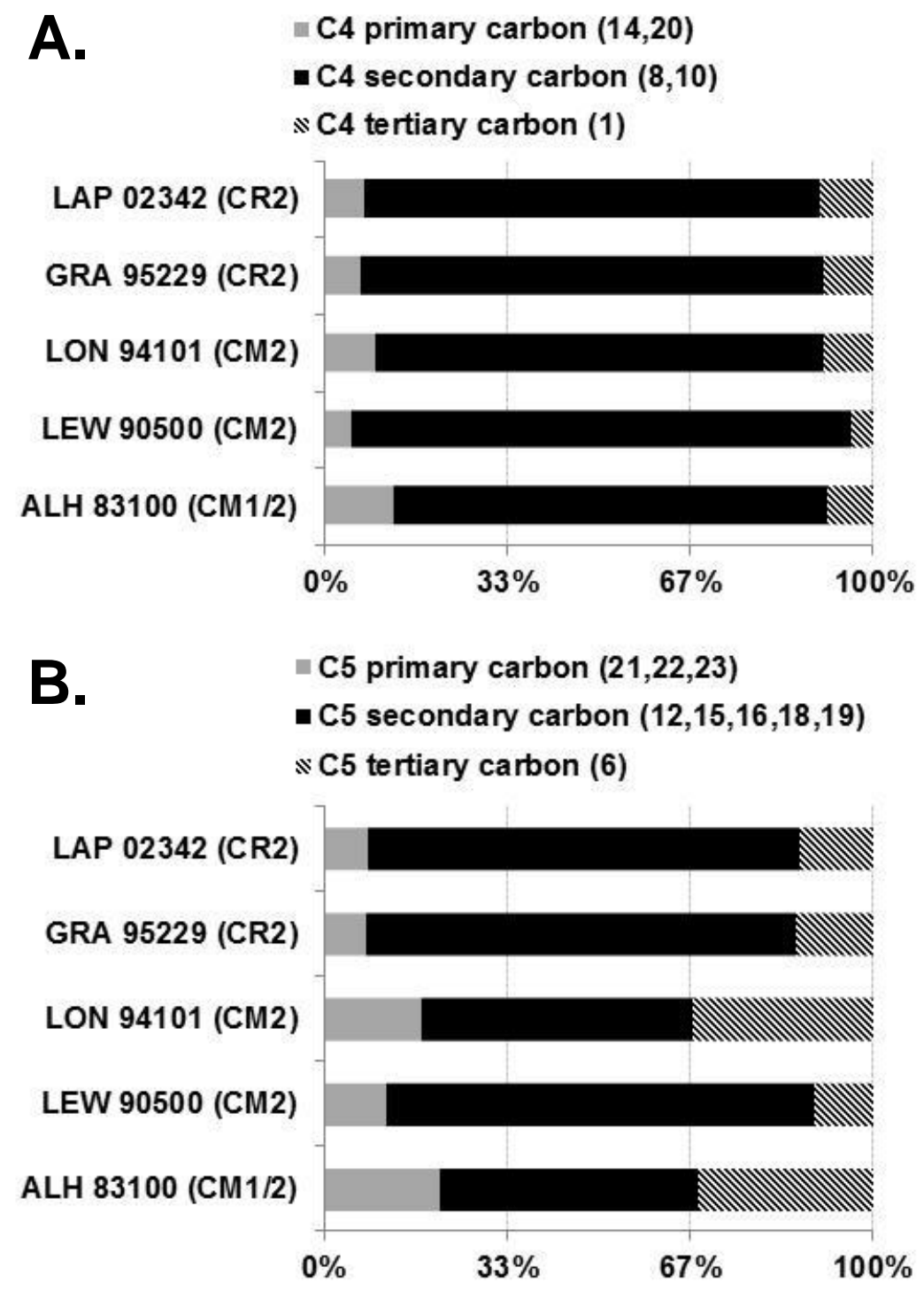


Figure 4. Relative abundances of $\mathrm{C}_{1}-\mathrm{C}_{4}$ amines and their structurally related amino acids (isobutylamine, tert-butylamine, and their corresponding structurally analogous amino acids were not included because of unknown abundances, see Table S2).
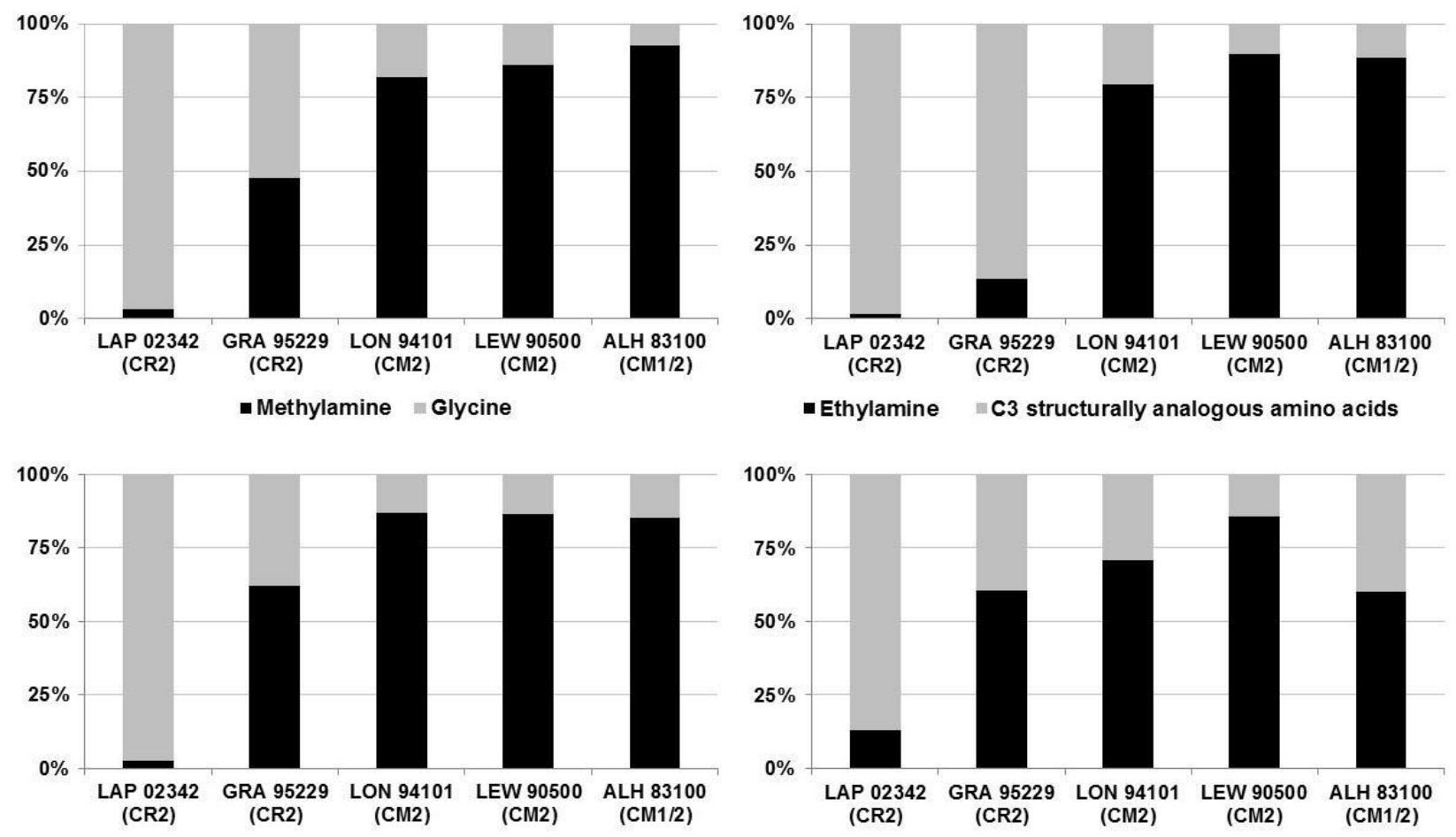

n-Propylamine $\quad$ C4 structurally analogous amino acids

- Isopropylamine $\quad$ C4 structurally analogous amino acids
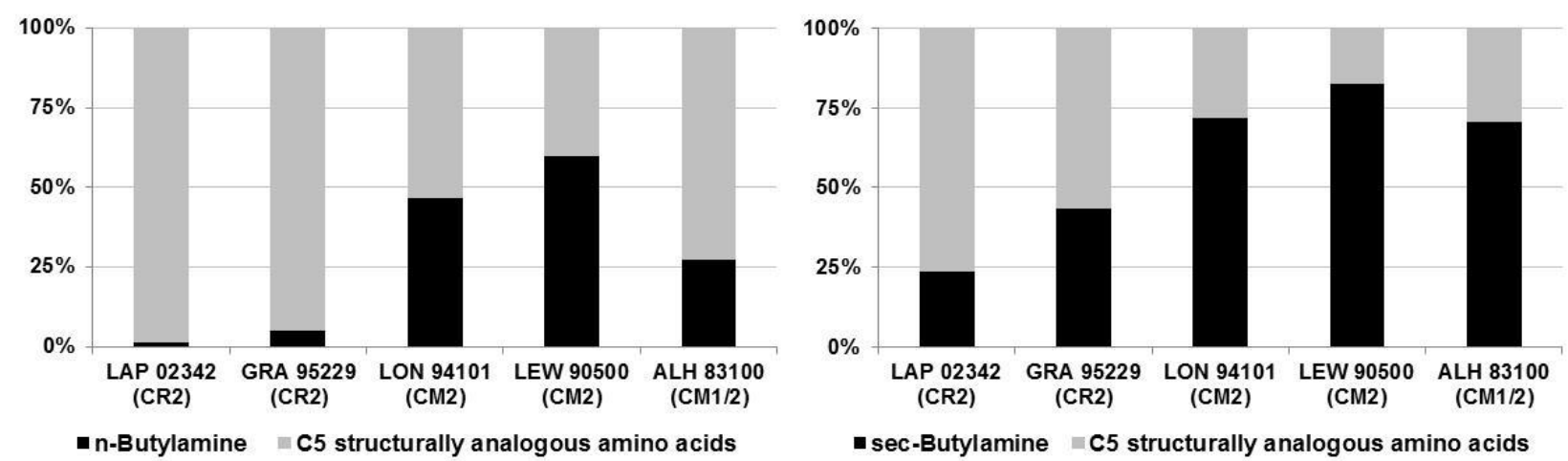

- sec-Butylamine $=$ C5 structurally analogous amino acids 
Figure 5. $\delta^{13} \mathrm{C}$ isotopic data for the amines measured from acid-hydrolyzed hot water extracts of CR2, CM2, and CM1/2 chondrites. Amines are grouped within each meteorite by carbon number. Meteorites are arranged in order of increasing aqueous alteration (Alexander et al., 2012, 2013).

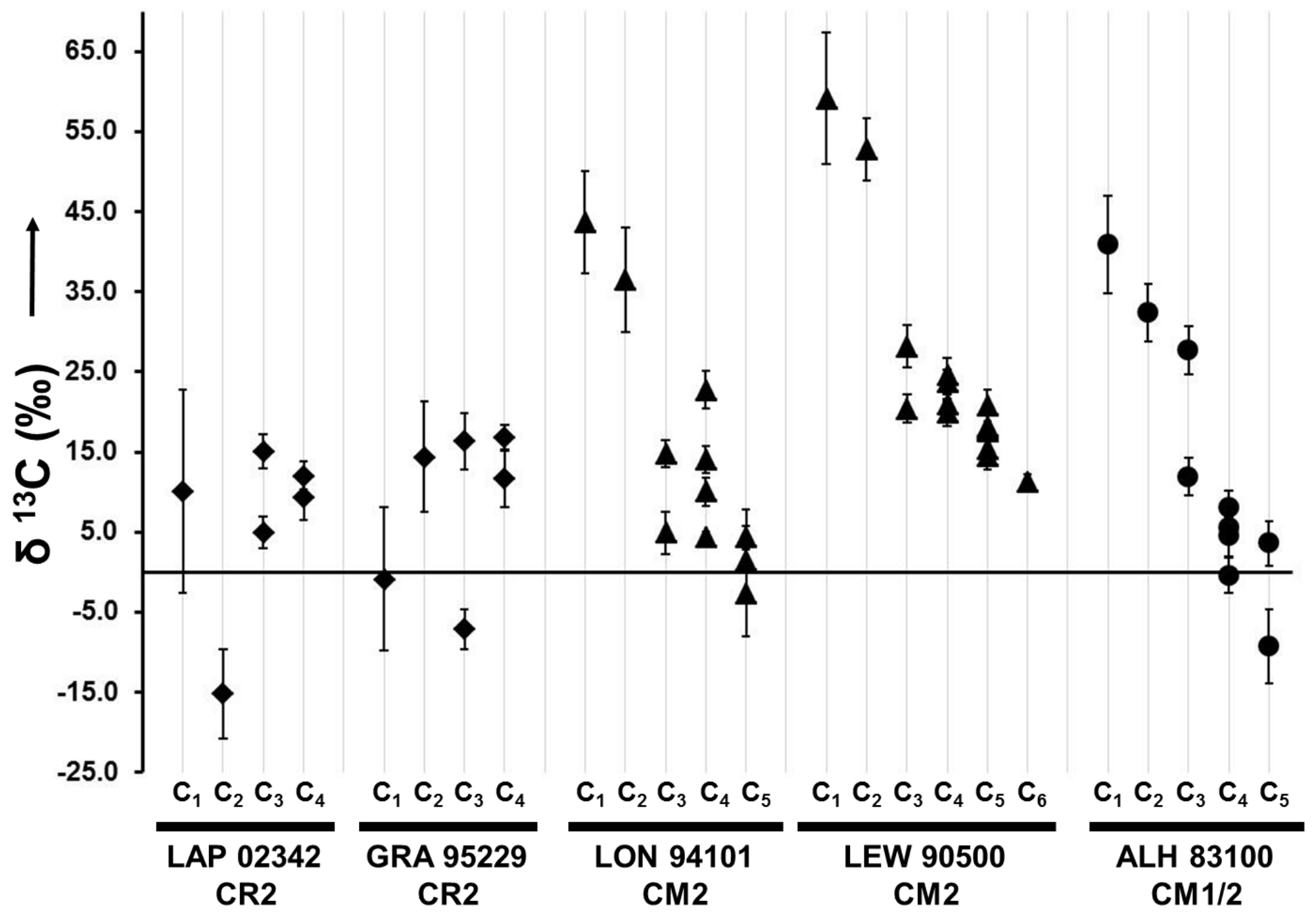


Figure 6. GC-MS chromatogram $(\mathrm{m} / \mathrm{z}=165.5-168.5)$ of $(R)$-sec-butylamine, diethylamine, and $(S)$-sec-butylamine (compounds $\mathbf{8}, 9$ and 10 respectively; U: unknown compound) in acidhydrolyzed hot water extracts of CR2, CM2, and CM1/2 chondrites and their corresponding racemic standards.
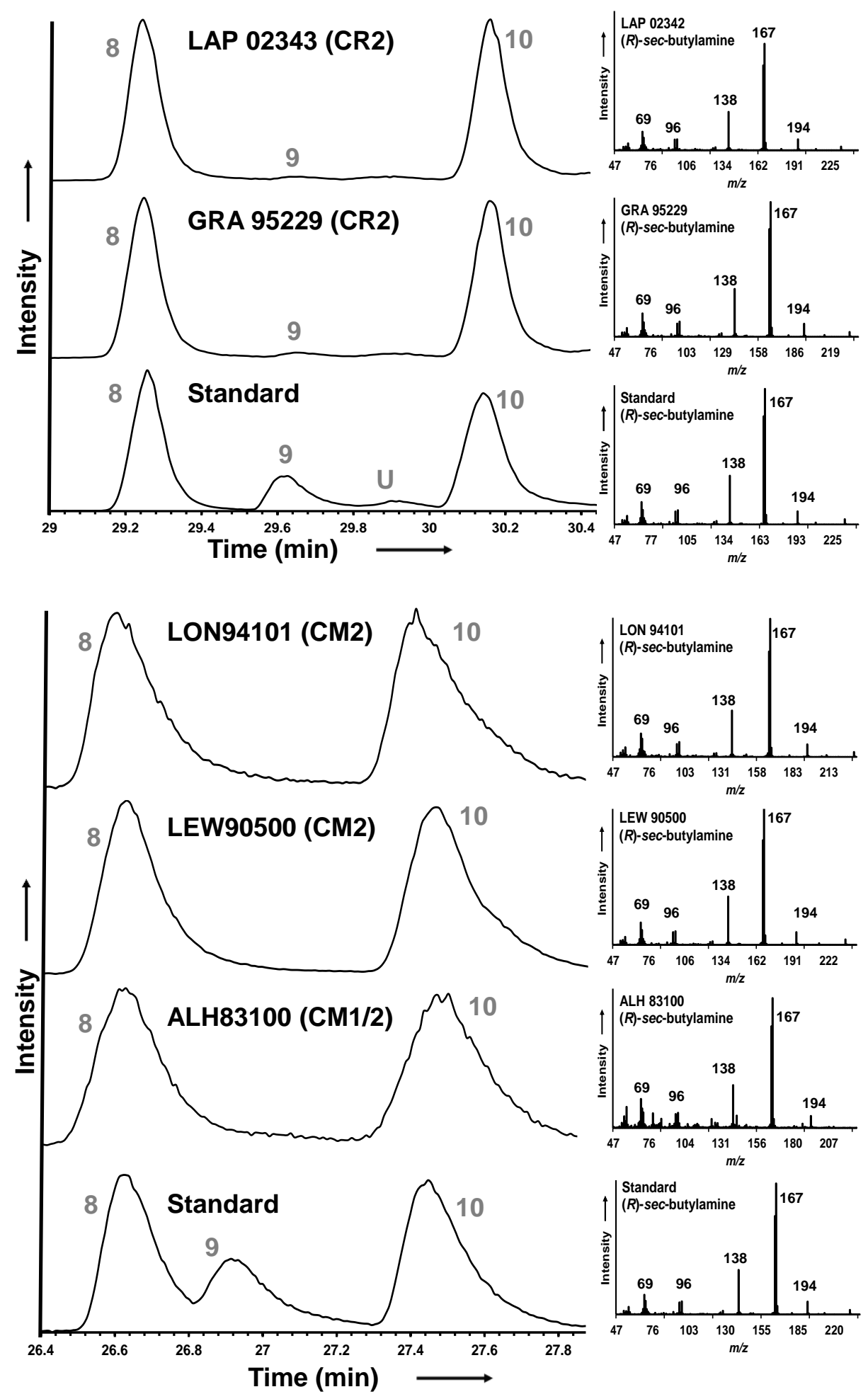
Table 1. Blank-corrected concentrations of amines in hot-water extracts of CR2, CM2, and CM1/2 chondrites; values in nmol/g of meteorite. ${ }^{\mathrm{a}}$

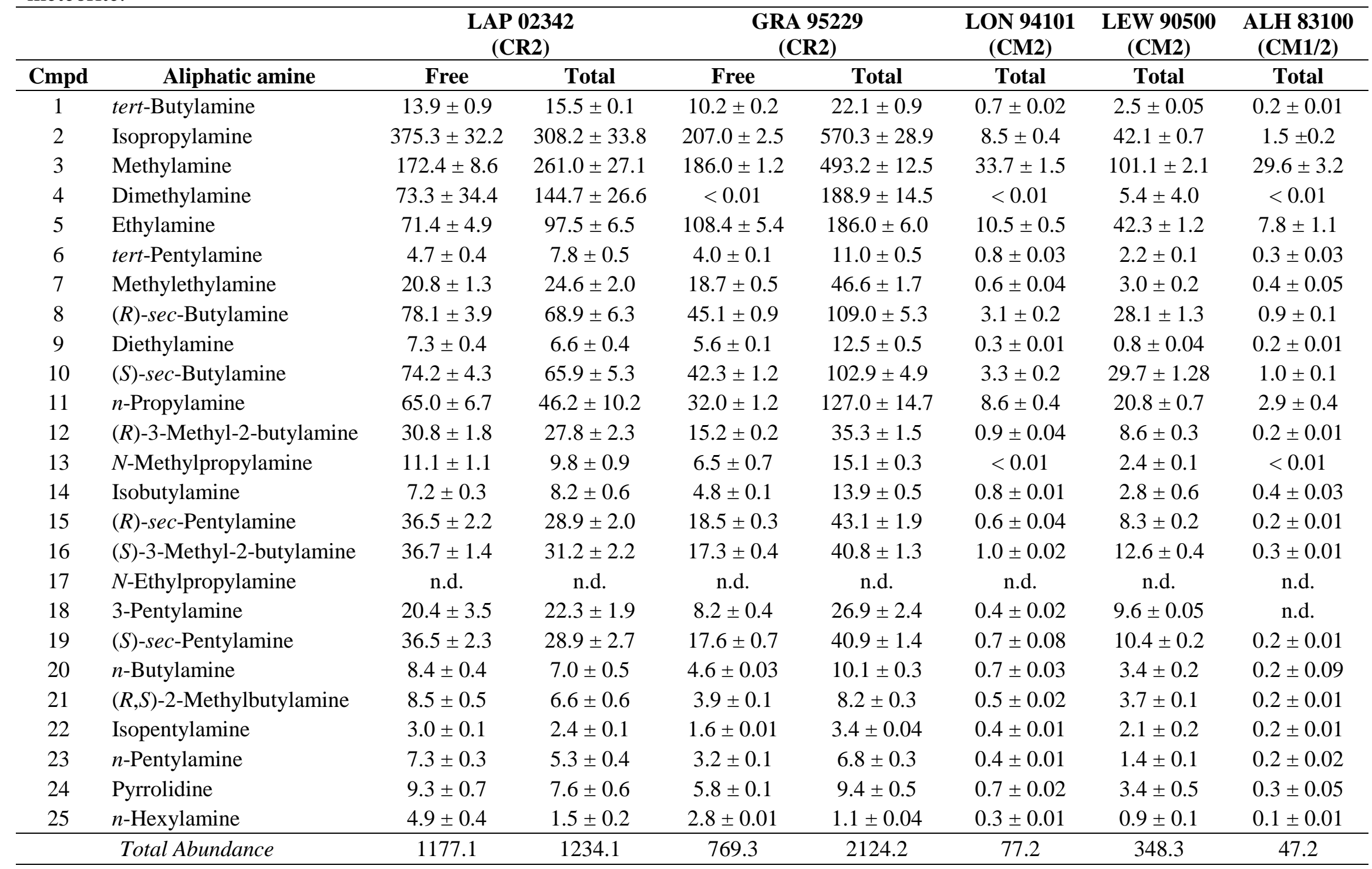

n.d.: Value could not be determined due to co-elution with $S$-TPC acid.

${ }^{a}$ Compounds identified by comparison with elution time and mass spectra of standards. Values are the average of three measurements from singleion gas chromatograms as detailed in Section 2.4; errors shown are standard deviations. 
Table 2. $\delta^{13} \mathrm{C}$ values (\%oVPDB) of amines (bold) and their structurally analogous amino acids from acid hydrolyzed hot-water extracts of CR2, CM2, and CM1/2 chondrites. ${ }^{\mathrm{a}}$

\begin{tabular}{|c|c|c|c|c|c|c|c|}
\hline \multirow[b]{2}{*}{ Compound } & \multicolumn{2}{|c|}{$\begin{array}{c}\text { LAP 02342 } \\
\text { (CR2) } \\
\end{array}$} & \multicolumn{2}{|c|}{$\begin{array}{c}\text { GRA 95229 } \\
\text { (CR2) } \\
\end{array}$} & \multirow{2}{*}{$\begin{array}{c}\text { LON } 94101 \\
(\text { CM2) } \\
\text { Total }\end{array}$} & \multirow{2}{*}{$\begin{array}{c}\text { LEW } 90500 \\
\text { (CM2) } \\
\text { Total }\end{array}$} & \multirow{2}{*}{$\begin{array}{c}\text { ALH 83100 } \\
(\text { CM1/2) }\end{array}$} \\
\hline & Free & Total & Free & Total & & & \\
\hline Methylamine $^{b}$ & n.d. & $10 \pm 13$ & n.d. & $-1 \pm 9$ & $44 \pm 6$ & $59 \pm 8$ & $41 \pm 6$ \\
\hline Glycine & n.a. & $20.1 \pm 0.1^{\mathrm{d}}$ & n.a. & $35 \pm 9^{e}$ & $36 \pm 4$ & $47 \pm 1$ & $53 \pm 3$ \\
\hline Ethylamine $^{\text {b }}$ & n.d. & $-15 \pm 6^{c}$ & n.d. & $14 \pm 7^{c}$ & $37 \pm 7$ & $53 \pm 4$ & $32 \pm 4$ \\
\hline D-Alanine & n.a. & $24.8 \pm 1.1^{\mathrm{d}}$ & n.a. & $40 \pm 3^{e}$ & $38 \pm 4$ & $25 \pm 4$ & $44 \pm 5$ \\
\hline L-Alanine & n.a. & $25.3 \pm 2.1^{\mathrm{d}}$ & n.a. & $38 \pm 2^{\mathrm{e}}$ & $41 \pm 1$ & $27 \pm 1$ & $35 \pm 3$ \\
\hline$\beta$-Alanine & n.a. & n.a. & n.a. & $8 \pm 20^{\mathrm{e}}$ & $17 \pm 4$ & $25 \pm 4$ & $4 \pm 4$ \\
\hline n-Propylamine & $-3 \pm 3^{d}$ & $5 \pm 2^{c}$ & $-0.2 \pm 2^{c}$ & $-7 \pm 3^{c}$ & $5 \pm 3$ & $20 \pm 2$ & $12 \pm 2$ \\
\hline D- $\alpha$-Aminobutyric acid & n.a. & n.a. & n.a. & $20 \pm 3^{\mathrm{e}}$ & $44 \pm 5^{\mathrm{e}}$ & n.d. & n.d. \\
\hline L- $\alpha$-Aminobutyric acid & n.a. & $3.5 \pm 1.5^{\mathrm{d}}$ & n.a. & $10 \pm 14^{\mathrm{e}}$ & n.d. & n.d. & n.d. \\
\hline$\gamma$-Aminobutyric acid & n.a. & n.a. & n.a. & $-18 \pm 11^{\mathrm{e}}$ & $19 \pm 3^{\mathrm{e}}$ & $17 \pm 10^{\mathrm{e}}$ & $-22 \pm 4^{\mathrm{e}}$ \\
\hline Isopropylamine $^{\text {b }}$ & $23 \pm 4^{d}$ & $15 \pm 2^{c}$ & $18 \pm 3^{c}$ & $16 \pm 4^{\mathrm{c}}$ & $15 \pm 2$ & $28 \pm 3$ & $28 \pm 3$ \\
\hline$\alpha$-Aminoisobutyric acid & n.a. & $11.2 \pm 0.2^{\mathrm{d}}$ & n.a. & $24 \pm 1^{\mathrm{e}}$ & $9 \pm 3$ & $22 \pm 3$ & n.d. \\
\hline$n$-Butylamine & n.d. & n.d. & n.d. & n.d. & $23 \pm 2$ & $20 \pm 2$ & $-0.4 \pm 2$ \\
\hline (R)-sec-Butylamine & $18 \pm 4$ & $9 \pm 3$ & $12 \pm 3$ & $12 \pm 4$ & $10 \pm 2$ & $25 \pm 2$ & $5 \pm 3$ \\
\hline (S)-sec-Butylamine & $11 \pm 4$ & $12 \pm 2$ & $9 \pm 4$ & $17 \pm 2$ & $14 \pm 2$ & $24 \pm 2$ & $8 \pm 2$ \\
\hline D,L-Isovaline & n.a. & $-5.1 \pm 1.5^{\mathrm{d}}$ & n.a. & $16 \pm 8^{\mathrm{e}}$ & $34 \pm 1$ & $34 \pm 1$ & n.a. \\
\hline $\mathrm{D}, \mathrm{L}-\delta$-Amino- $n$-valeric acid & n.a. & n.a. & n.a. & n.a. & $-12 \pm 9^{\mathrm{e}}$ & n.a. & $-2 \pm 4^{\mathrm{e}}$ \\
\hline tert-Butylamine & n.d. & n.d. & n.d. & n.d. & $4 \pm 1$ & $2 \pm 0.3$ & $6 \pm 1$ \\
\hline$n$-Pentylamine & n.d. & n.d. & n.d. & n.d. & n.d. & $15 \pm 2$ & n.d. \\
\hline (S)-sec-Pentylamine & n.d. & n.d. & n.d. & n.d. & $4 \pm 3$ & $15 \pm 3$ & n.d. \\
\hline$(R, S)$-2-Methylbutylamine & n.d. & n.d. & n.d. & n.d. & $2 \pm 4$ & $18 \pm 1$ & $-9 \pm 5$ \\
\hline Isopentylamine & n.d. & n.d. & n.d. & n.d. & n.d. & $18 \pm 2$ & n.d. \\
\hline tert-Pentylamine & n.d. & n.d. & n.d. & n.d. & $-3 \pm 6$ & $21 \pm 2$ & $4 \pm 3$ \\
\hline 3-Pentylamine & n.d. & n.d. & n.d. & n.d. & n.d. & $18 \pm 2$ & n.d. \\
\hline n-Hexylamine & n.d. & n.d. & n.d. & n.d. & n.d. & $11 \pm 1$ & n.d. \\
\hline Dimethylamine $^{b}$ & n.d. & $-11 \pm 7^{c}$ & n.d. & $-15 \pm 7^{c}$ & $-3 \pm 9^{c}$ & $-0.1 \pm 4^{c}$ & $-38 \pm 3^{c}$ \\
\hline
\end{tabular}

n.d.: Value could not be determined due to co-elution (caprolactam, phthalate, or $S$-TPC reagent) or complex mixture of compounds. n.a.: Not available in the literature.

${ }^{a}$ Values are the average of three measurements; errors shown are standard deviations.

${ }^{\mathrm{b}}$ Methylamine partially co-elutes with isopropylamine; and ethylamine partially co-elutes with dimethylamine.

${ }^{c}$ Contains a contribution from the amines in the derivatization reagent.

${ }^{\mathrm{d}}$ Values from Pizzarello and Holmes, 2009.

'Values from Elsila et al., 2012. 
Table 3. Enantiomeric ratios and enantiomeric excess for chiral amines $(R / S)$ extracted from CR2, CM2, and CM1/2 carbonaceous chondrites. ${ }^{\mathrm{a}}$

\begin{tabular}{lcccc}
\hline Sample & Isovaline & $\begin{array}{c}\text { Sec- } \\
\text { butylamine }\end{array}$ & $\begin{array}{c}\text { 3-Methyl-2- } \\
\text { butylamine }\end{array}$ & Sec-pentylamine \\
\hline & $(\mathbf{D} / \mathbf{L})$ & $(\boldsymbol{R} / \boldsymbol{S})^{\mathrm{a}}$ & $(\boldsymbol{R} / \boldsymbol{S})^{\mathrm{a}}$ & $(\boldsymbol{R} / \boldsymbol{S})^{\mathrm{a}}$ \\
\hline LAP 02342 (CR2) Free & n.a. & $0.99 \pm 0.01$ & $0.99 \pm 0.02$ & $0.96 \pm 0.04$ \\
LAP 02342 (CR2) Total & n.a. & $0.99 \pm 0.01$ & $1.00 \pm 0.04$ & $0.97 \pm 0.05$ \\
GRA 95229 (CR2) Free & n.a. & $1.00 \pm 0.01$ & $0.98 \pm 0.03$ & $0.99 \pm 0.03$ \\
GRA 95229 (CR2) Total & n.a. & $1.00 \pm 0.01$ & $0.99 \pm 0.05$ & $0.97 \pm 0.05$ \\
LON 94101 (CM2) Total & $0.96 \pm 0.05$ & $0.99 \pm 0.02$ & $0.97 \pm 0.03$ & $0.96 \pm 0.06$ \\
LEW 90500 (CM2) Total & $1.01 \pm 0.07$ & $0.98 \pm 0.03$ & $0.99 \pm 0.02$ & $0.97 \pm 0.04$ \\
ALH 83100 (CM1/2) Total & $0.87 \pm 0.09$ & $0.99 \pm 0.02$ & $0.97 \pm 0.04$ & $0.97 \pm 0.04$ \\
\hline
\end{tabular}

n.a.: Not available in the literature.

${ }^{a}$ Values have been corrected against injections of racemic standards to account for instrument response. Enantiomeric ratios and standard deviations of amines are based on three separate measurements from single ion gas chromatograms extracted at $\mathrm{m} / \mathrm{z}=165.5-168.5$ for $\mathrm{sec}$ butylamine, and at $\mathrm{m} / \mathrm{z}=235.5-238.5$ for 3-methyl-2-butylamine and sec-pentylamine. Enantiomeric ratios and standard deviations of isovaline are based on three separate measurements from single ion LC-FD/ToF-MS chromatograms extracted at $\mathrm{m} / z=379.13 \pm$ 0.015 in positive electrospray ionization mode. 


\section{Supporting Information}

\section{Aliphatic Amines in Antarctic CR2, CM2, and CM1/2 Carbonaceous Chondrites}

José C. Aponte, ${ }^{\mathrm{a}, \mathrm{b}, *}$ Hannah L. McLain, ${ }^{\text {a,b }}$ Jason P. Dworkin, ${ }^{\mathrm{a}}$ and Jamie E. Elsila ${ }^{\mathrm{a}}$

a Solar System Exploration Division, Code 691, NASA Goddard Space Flight Center, Greenbelt, Maryland 20771, USA.

${ }^{\mathrm{b}}$ Department of Chemistry, Catholic University of America, Washington, DC 20064, USA.

* Corresponding author:

jose.c.aponte@ @asa.gov; tel.: +1.301.614.6916; fax: +1.301.286.1683. 
Figure S1. Structures of aliphatic amines listed in Table 1.

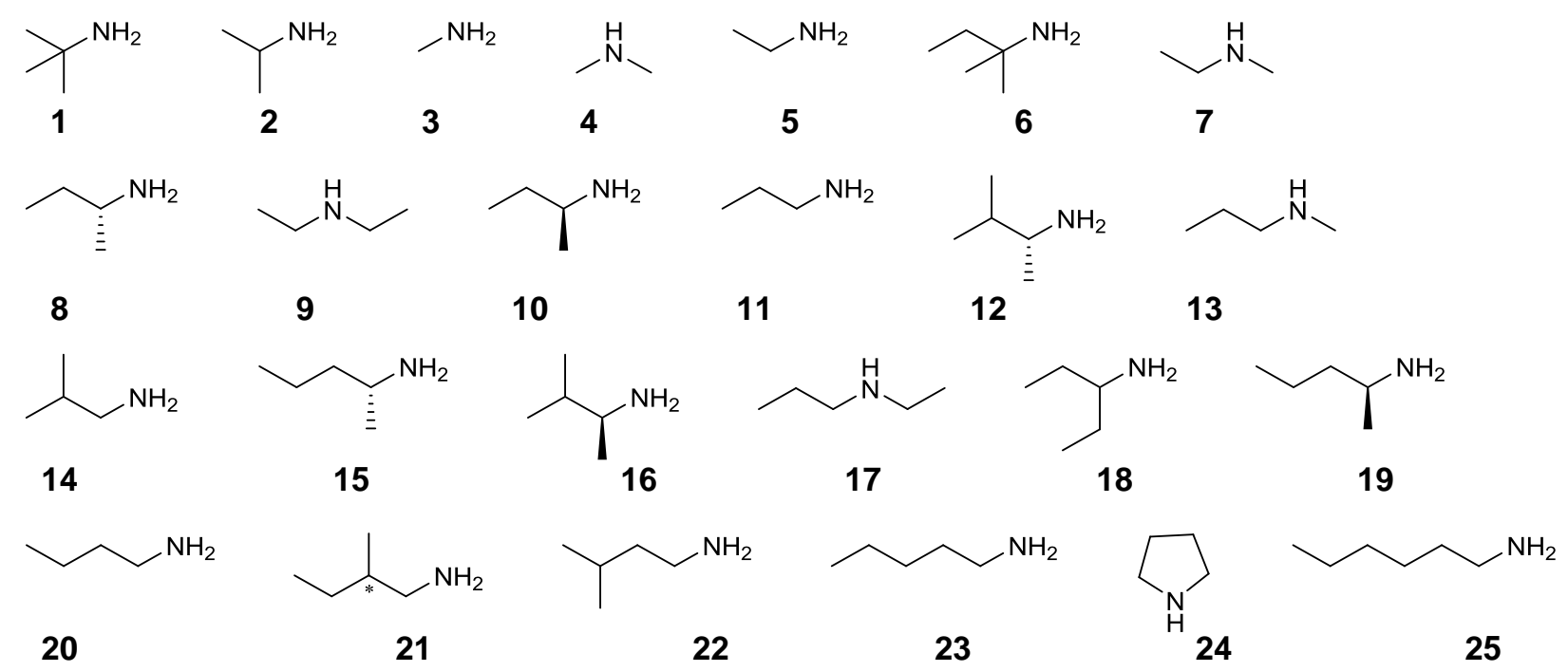


Figure S2. Structures of amines and amino acids sharing the same aliphatic backbones.

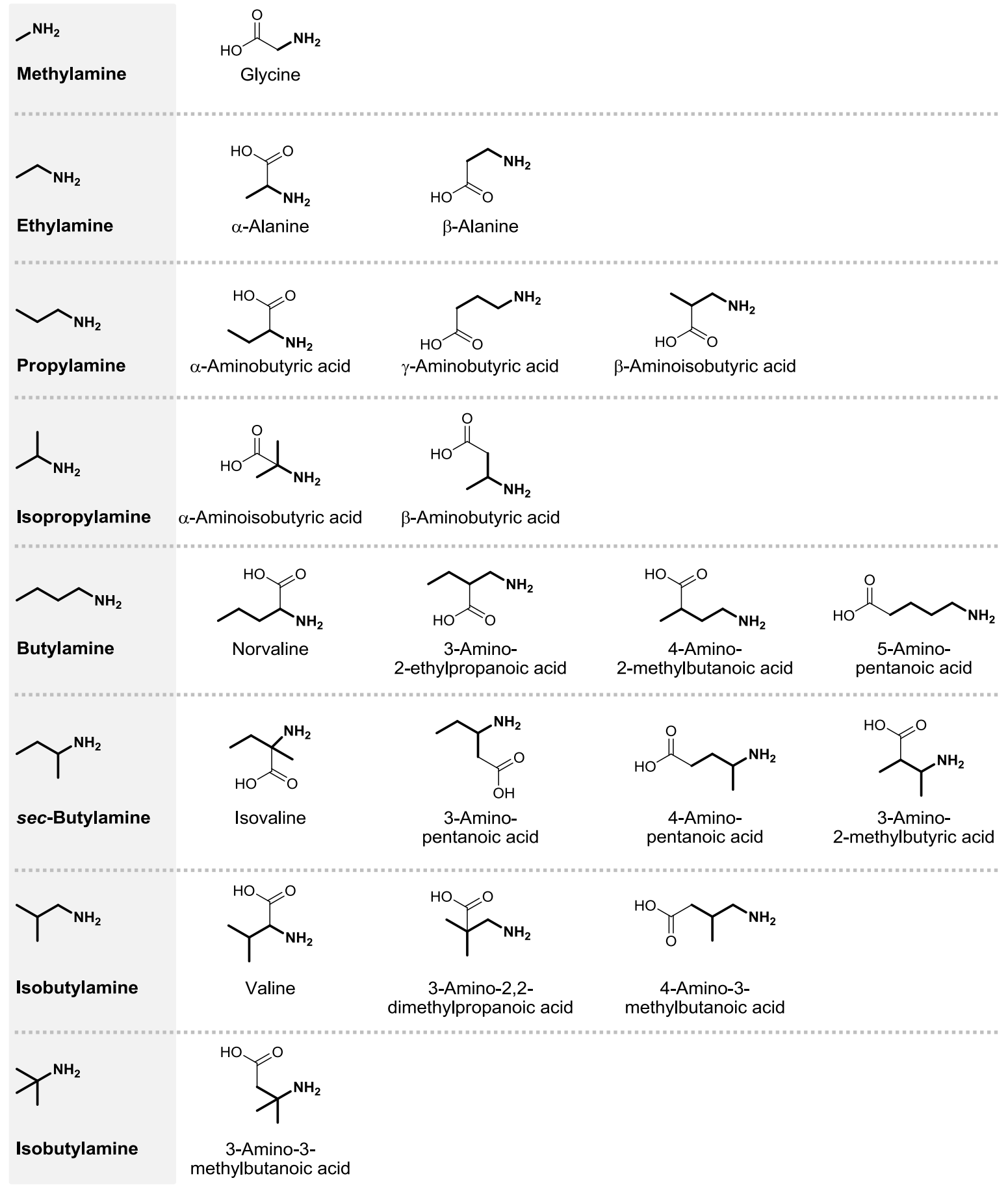


Table S1. Summary of the total average blank-corrected amino acid concentrations (nmol/g of meteorite) of the two- to six-carbon amino acids identified in the acid-hydrolyzed, hot-water extracts of CM2 and CM1/2 carbonaceous chondrites. ${ }^{\text {a }}$

\begin{tabular}{|c|c|c|c|}
\hline Amino Acid & LON 94101 & LEW 90500 & ALH 83100 \\
\hline D-aspartic acid & $<0.01$ & $0.06 \pm 0.08$ & $<0.01$ \\
\hline L-aspartic acid & $<0.01$ & $<0.01$ & $0.02 \pm 0.02$ \\
\hline D-glutamic acid & n.d. & n.d. & n.d. \\
\hline L-glutamic acid & $0.3 \pm 0.08$ & $0.3 \pm 0.08$ & $0.02 \pm 0.03$ \\
\hline D-serine & $0.01 \pm 0.003$ & $0.02 \pm 0.01$ & $<0.01$ \\
\hline L-serine & $0.02 \pm 0.01$ & $0.02 \pm 0.01$ & $<0.01$ \\
\hline Glycine & $4.9 \pm 1.6$ & $10.1 \pm 0.3$ & $1.9 \pm 0.3$ \\
\hline D-alanine & $0.9 \pm 0.2$ & $1.8 \pm 0.04$ & $0.1 \pm 0.05$ \\
\hline L-alanine & $0.6 \pm 0.2$ & $1.4 \pm 0.1$ & $0.2 \pm 0.04$ \\
\hline$\beta$-alanine (BALA) & $1.2 \pm 0.4$ & $1.7 \pm 0.05$ & $0.7 \pm 0.1$ \\
\hline $\mathrm{D}, \mathrm{L}-\alpha$-amino- $n$-butyric acid (D,L- $\alpha-\mathrm{ABA})$ & $0.5 \pm 0.1$ & $1.0 \pm 0.04$ & $0.1 \pm 0.03$ \\
\hline D- $\beta$-amino- $n$-butyric acid (D- $\beta$-ABA) & $1.0 \pm 0.3$ & $1.3 \pm 0.03$ & $0.3 \pm 0.04$ \\
\hline L- $\beta$-amino- $n$-butyric acid (L- $\beta$-ABA) & $1.5 \pm 0.4$ & $4.6 \pm 0.2$ & $0.4 \pm 0.1$ \\
\hline$\gamma$-amino butyric acid (GABA) & $0.8 \pm 0.3$ & $2.2 \pm 0.04$ & $0.4 \pm 0.04$ \\
\hline$\alpha$-aminoisobutyric acid $(\alpha$-AIB) & $1.0 \pm 0.3$ & $1.2 \pm 0.03$ & $0.3 \pm 0.03$ \\
\hline$\varepsilon$-amino- $n$-caproic acid $(\mathrm{EACA})^{\mathrm{b}}$ & $1.8 \pm 0.4$ & $1.7 \pm 0.2$ & $1.0 \pm 0.1$ \\
\hline D-2-aminopentanoic acid (D-norvaline) & n.d. & $<0.01$ & $<0.01$ \\
\hline L-2-aminopentanoic acid (L-norvaline) & n.d. & $<0.01$ & $<0.01$ \\
\hline D-2-amino-2-methylbutanoic acid (D-isovaline) & $0.6 \pm 0.02$ & $4.8 \pm 1.0$ & $0.2 \pm 0.04$ \\
\hline L-2-amino-2-methylbutanoic acid (L-isovaline) & $0.7 \pm 0.05$ & $4.8 \pm 1.0$ & $0.2 \pm 0.04$ \\
\hline D-2-amino-3-methylbutanoic acid (D-valine) & $0.1 \pm 0.02$ & $0.2 \pm 0.02$ & $0.02 \pm 0.001$ \\
\hline L-2-amino-3-methylbutanoic acid (L-valine) & $0.1 \pm 0.01$ & $0.2 \pm 0.01$ & $0.02 \pm 0.003$ \\
\hline D,L-3-aminopentanoic acid (D,L-3-apa) & $0.3 \pm 0.01$ & $0.6 \pm 0.04$ & $0.3 \pm 0.04$ \\
\hline $\begin{array}{l}\mathrm{D}, \mathrm{L}-\text { and allo-3-amino-2-methylbutanoic acid (D,L- and } \\
\text { allo-3-a-2-mba) }\end{array}$ & $0.2 \pm 0.01$ & n.d. & n.d. \\
\hline 3-amino-3-methylbutanoic acid (3-a-3-mba) & $0.4 \pm 0.04$ & $0.1 \pm 0.01$ & $0.2 \pm 0.02$ \\
\hline 3-amino-2,2-dimethylpropanoic acid (3-a-2,2-dmpa) & $0.2 \pm 0.01$ & $0.8 \pm 0.1$ & $0.2 \pm 0.03$ \\
\hline D,L-3-amino-2-ethylpropanoic acid (D,L-3-a-2-epa) & $0.1 \pm 0.01$ & $0.1 \pm 0.01$ & $0.04 \pm 0.01$ \\
\hline D,L-4-aminopentanoic acid (D,L-4-apa) & $0.7 \pm 0.03$ & $2.2 \pm 0.05$ & $0.1 \pm 0.01$ \\
\hline D,L-4-amino-2-methylbutanoic acid (D,L-4-a-2-mba) & $0.3 \pm 0.03$ & $0.8 \pm 0.2$ & $0.2 \pm 0.02$ \\
\hline D,L-4-amino-3-methylbutanoic acid (D,L-4-a-3-mba) & $0.1 \pm 0.01$ & $0.3 \pm 0.03$ & $0.1 \pm 0.01$ \\
\hline 5-aminopentanoic acid (5-apa) & $0.4 \pm 0.03$ & $1.4 \pm 0.1$ & $0.3 \pm 0.01$ \\
\hline
\end{tabular}

n.d.: Not determined.

${ }^{a}$ Extracts were analyzed by OPA/NAC derivatization (1 and $15 \mathrm{~min}$ ) and UPLC separation with UV fluorescence and time of flight mass spectrometry (ToF-MS) detection. For the LC-ToF-MS data, the mono-isotopic masses of each protonated OPA/NAC amino acid derivative $(\mathrm{M}+\mathrm{H}+)$ was used for quantification and final peak integrations included background level correction using a serpentine blank and a comparison of the peak areas with those of an amino acid standard run on the same day. The uncertainties $(\delta x)$ are based on the standard deviation of the average value of 3 separate measurements $(n)$.

becomposition of Nylon-6 may contribute to the abundances (e.g Glavin et al. 2006). 
Table S2. Concentration of meteoritic amines (bold) and their structurally related amino acids in acidhydroyzed hot-water extracts; values expressed in nmol/g of meteorite.

\begin{tabular}{|c|c|c|c|c|c|}
\hline Compound & $\begin{array}{l}\text { LAP 02342 } \\
\text { (CR2) }\end{array}$ & $\begin{array}{l}\text { GRA } 95229 \\
\text { (CR2) }\end{array}$ & $\begin{array}{l}\text { LON } 94101 \\
\text { (CM2) }\end{array}$ & $\begin{array}{l}\text { LEW } 90500 \\
\text { (CM2) }\end{array}$ & $\begin{array}{l}\text { ALH 83100 } \\
\text { (CM1/2) }\end{array}$ \\
\hline Methylamine & $261.0 \pm 27.1$ & $493.2 \pm 12.5$ & $33.7 \pm 1.5$ & $101.1 \pm 2.1$ & $29.6 \pm 3.2$ \\
\hline Glycine & $8268^{a}$ & $539.9 \pm 13.7^{b}$ & $7.4 \pm 3.4$ & $16.3 \pm 6.8$ & $2.3 \pm 0.6$ \\
\hline Ethylamine & $97.5 \pm 6.5$ & $186.0 \pm 6.0$ & $10.5 \pm 0.5$ & $42.3 \pm 1.2$ & $7.8 \pm 1.1$ \\
\hline D-Alanine & \multirow{2}{*}{$5486^{\mathrm{a}}$} & $589.5 \pm 77.1^{b}$ & $0.9 \pm 0.2$ & $1.8 \pm 0.04$ & $0.1 \pm 0.05$ \\
\hline L-Alanine & & $574.6 \pm 70.5^{\mathrm{b}}$ & $0.6 \pm 0.2$ & $1.4 \pm 0.1$ & $0.2 \pm 0.04$ \\
\hline$\beta$-Alanine & $129^{\mathrm{a}}$ & $35.3 \pm 5.6^{\mathrm{b}}$ & $1.2 \pm 0.4$ & $1.7 \pm 0.05$ & $0.7 \pm 0.1$ \\
\hline$n$-Propylamine & $46.2 \pm 10.2$ & $127.0 \pm 14.7$ & $8.6 \pm 0.4$ & $20.8 \pm 0.7$ & $2.9 \pm 0.4$ \\
\hline D,L- $\alpha$-Aminobutyric acid & $1367^{\mathrm{a}}$ & $57.4 \pm 2.4^{\mathrm{b}}$ & $0.5 \pm 0.1$ & $1.0 \pm 0.04$ & $0.1 \pm 0.03$ \\
\hline$\gamma$-Aminobutyric acid & $317^{\mathrm{a}}$ & $18.6 \pm 3.9^{\mathrm{b}}$ & $0.8 \pm 0.3$ & $2.2 \pm 0.04$ & $0.4 \pm 0.04$ \\
\hline$\beta$-Aminoisobutyric acid & $56^{\mathrm{a}}$ & $2.0 \pm 3.4^{\mathrm{b}}$ & n.d. & n.d. & n.d. \\
\hline Isopropylamine & $308.2 \pm 33.8$ & $570.3 \pm 28.9$ & $8.5 \pm 0.4$ & $42.1 \pm 0.7$ & $1.5 \pm 0.2$ \\
\hline$\alpha$-Aminoisobutyric acid & $1897^{\mathrm{a}}$ & $293.8 \pm 11.9^{b}$ & $1.0 \pm 0.3$ & $1.2 \pm 0.03$ & $0.3 \pm 0.03$ \\
\hline D- $\beta$-Aminobutyric acid & \multirow{2}{*}{$203^{\mathrm{a}}$} & $37.9 \pm 3.7^{\mathrm{b}}$ & $1.0 \pm 0.3$ & $1.3 \pm 0.03$ & $0.3 \pm 0.04$ \\
\hline L- $\beta$-Aminobutyric acid & & $41.2 \pm 4.8^{\mathrm{b}}$ & $1.5 \pm 0.4$ & $4.6 \pm 0.2$ & $0.4 \pm 0.1$ \\
\hline$n$-Butylamine & $7.0 \pm 0.5$ & $10.1 \pm 0.3$ & $0.7 \pm 0.03$ & $3.4 \pm 0.2$ & $0.2 \pm 0.09$ \\
\hline D-Norvaline & \multirow{2}{*}{$417^{\mathrm{a}}$} & \multirow{2}{*}{$184^{\mathrm{a}}$} & n.d. & $<0.01$ & $<0.01$ \\
\hline L-Norvaline & & & n.d. & $<0.01$ & $<0.01$ \\
\hline $\begin{array}{l}\text { D,L-3-amino-2- } \\
\text { ethylpropanoic acid }\end{array}$ & n.a. & n.a. & $0.1 \pm 0.01$ & $0.1 \pm 0.01$ & $0.04 \pm 0.01$ \\
\hline $\begin{array}{l}\text { D,L-4-amino-2- } \\
\text { methylbutanoic acid }\end{array}$ & n.a. & n.a. & $0.3 \pm 0.03$ & $0.8 \pm 0.2$ & $0.2 \pm 0.02$ \\
\hline D,L-5-Aminopentanoic acid & $87^{\mathrm{a}}$ & $12^{\mathrm{a}}$ & $0.4 \pm 0.03$ & $1.4 \pm 0.1$ & $0.3 \pm 0.01$ \\
\hline sec-Butylamine & $134.8 \pm 8.2$ & $211.9 \pm 7.2$ & $6.4 \pm 0.3$ & $57.8 \pm 1.8$ & $1.9 \pm 0.1$ \\
\hline D-Isovaline & $203^{\mathrm{a}}$ & $2407+10^{b}$ & $0.6 \pm 0.02$ & $4.8 \pm 1.0$ & $0.2 \pm 0.02$ \\
\hline L-Isovaline & $293^{a}$ & $249.1 \pm 19^{\circ}$ & $0.7 \pm 0.05$ & $4.8 \pm 1.0$ & $0.2 \pm 0.02$ \\
\hline D,L-3-Aminopentanoic acid & $23^{\mathrm{a}}$ & $7^{\mathrm{a}}$ & $0.3 \pm 0.01$ & $0.6 \pm 0.04$ & $0.3 \pm 0.04$ \\
\hline D,L-4-Aminopentanoic acid & $119^{\mathrm{a}}$ & $23^{\mathrm{a}}$ & $0.7 \pm 0.03$ & $2.2 \pm 0.05$ & $0.1 \pm 0.01$ \\
\hline $\begin{array}{l}\text { D,L- and allo-3-Amino-2- } \\
\text { methylbutyric acid }\end{array}$ & n.a. & n.a. & $0.2 \pm 0.01$ & n.d. & n.d. \\
\hline Isobutylamine & $8.2 \pm 0.6$ & $13.9 \pm 0.5$ & $0.8 \pm 0.01$ & $2.8 \pm 0.6$ & $0.4 \pm 0.03$ \\
\hline D-Valine & & $59.7 \pm 6.0^{\mathrm{b}}$ & $0.1 \pm 0.02$ & $0.2 \pm 0.02$ & $0.02 \pm 0.001$ \\
\hline L-Valine & $800^{\mathrm{a}}$ & $61.1 \pm 6.7^{b}$ & $0.1 \pm 0.01$ & $0.2 \pm 0.01$ & $0.02 \pm 0.003$ \\
\hline $\begin{array}{l}\text { 3-Amino-2,2- } \\
\text { dimethylpropanoic acid }\end{array}$ & n.a. & n.a. & $0.2 \pm 0.01$ & $0.8 \pm 0.1$ & $0.2 \pm 0.03$ \\
\hline $\begin{array}{l}\text { 4-amino-3-methylbutanoic } \\
\text { acid }\end{array}$ & $0.8^{\mathrm{a}}$ & $0.1^{\mathrm{a}}$ & $0.1 \pm 0.01$ & $0.3 \pm 0.03$ & $0.1 \pm 0.01$ \\
\hline tert-Butylamine & $15.5 \pm 0.1$ & $22.1 \pm 0.9$ & $0.7 \pm 0.02$ & $2.5 \pm 0.05$ & $0.2 \pm 0.01$ \\
\hline $\begin{array}{l}\text { 3-Amino-3-methylbutanoic } \\
\text { acid }\end{array}$ & n.a. & n.a. & $0.4 \pm 0.04$ & $0.1 \pm 0.01$ & $0.2 \pm 0.02$ \\
\hline
\end{tabular}

n.a.: Not available in the literature. n.d.: Not determined.

aPizzarello S. and Holmes W. (2009) Geochim. Cosmochim. Acta 73, 2150-2162.

${ }^{\mathrm{b}}$ Martins Z. et al. (2007) Meteorit. Planet. Sci. 42, 2125-2136. 
Table S3. Summary of the $\delta^{13} \mathrm{C}$ values (\% VPDB) of amino acids in CM2 and CM1/2 carbonaceous chondrites. $^{\mathrm{a}}$

\begin{tabular}{lccc}
\hline Amino Acid & ALH83100 & LON 94101 & LEW90500 \\
\hline Glycine & $53.3 \pm 2.6^{\mathrm{c}}$ & $35.5 \pm 3.6$ & $47.1 \pm 3.4$ \\
D-alanine & $16.0 \pm 4.6$ & $38.3 \pm 3.8$ & $25.1 \pm 3.7^{\mathrm{c}}$ \\
L-alanine & $34.8 \pm 2.9$ & $40.6 \pm 1.0$ & $27.3 \pm 1.2$ \\
$\beta$-alanine & $3.6 \pm 4.4$ & $17.3 \pm 4.1$ & $24.6 \pm 4.0$ \\
$\alpha$-AIB & n.d. & $8.9 \pm 3.0$ & $21.8 \pm 2.9$ \\
D,L-isovaline & n.d. & $34.2 \pm 0.8$ & $22.2 \pm 0.5$ \\
L-glutamic acid & n.d. & $27.0 \pm 2.0$ & $25.3 \pm 4.1$ \\
EACA & $-27.2 \pm 1.4$ & $-24.5 \pm 1.6$ & $-25.6 \pm 2.2$ \\
\hline
\end{tabular}

${ }^{a}$ Values are the average of three measurements; errors shown are standard deviations.

'Includes small contribution from unidentified coeluting peak.

n.d: Value could not be determined due to coeluting compounds or insufficient abundance. 